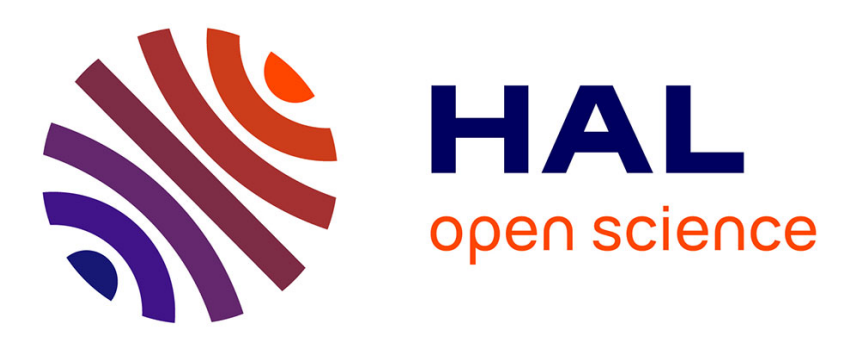

\title{
Global and local estimates for nonlinear noncoercive elliptic equations with measure data \\ Jerome Droniou
}

\section{To cite this version:}

Jerome Droniou. Global and local estimates for nonlinear noncoercive elliptic equations with measure data. Communications in Partial Differential Equations, 2003, 28, pp.no 1-2, 129-153. 10.1081/PDE120019377 . hal-00003935

\section{HAL Id: hal-00003935 \\ https://hal.science/hal-00003935}

Submitted on 19 Jan 2005

HAL is a multi-disciplinary open access archive for the deposit and dissemination of scientific research documents, whether they are published or not. The documents may come from teaching and research institutions in France or abroad, or from public or private research centers.
L'archive ouverte pluridisciplinaire HAL, est destinée au dépôt et à la diffusion de documents scientifiques de niveau recherche, publiés ou non, émanant des établissements d'enseignement et de recherche français ou étrangers, des laboratoires publics ou privés. 
CPDE, ?(?), 1-32 (?)

\title{
Global and local estimates for nonlinear noncoercive elliptic equations with measure data
}

\author{
Jérôme Droniou \\ Département de Mathématiques, CC 051, \\ Université Montpellier II, Place Eugène Bataillon, \\ 34095 Montpellier Cedex 5, France. Email: \\ droniou@math. univ-montp2.fr
}

\begin{abstract}
We study nonlinear noncoercive elliptic problems with measure data, proving first that the global estimates already known when the problem is coercive are still true for noncoercive problems. We then prove new estimates, on sets far from the support of the singular part of the right-hand side, in the energy space associated to the operator, which entails additional regularity results on the solutions.
\end{abstract}

Key Words: Non-linear elliptic equations, measures, non-coercivity, local estimates. 


\section{Introduction and main results}

\section{A. The problem}

Let $\Omega$ be a bounded open subset of $\mathbb{R}^{N}$. The purpose of this work is to obtain global and local estimates on the weak solutions to:

$$
\begin{cases}-\operatorname{div}(a(x, u, \nabla u))-\operatorname{div}(\Phi(x, u))=\mu+f & \text { in } \Omega, \\ u=0 & \text { on } \partial \Omega\end{cases}
$$

where $-\operatorname{div}(a(x, u, \nabla u))$ is a Leray-Lions operator on $W_{0}^{1, p}(\Omega)(1<$ $p \leq N), \Phi(x, u)$ is a convection term with growth properties, $f \in$ $W^{-1, p^{\prime}}(\Omega)$ and $\mu \in \mathcal{M}(\Omega)$ (see the precise hypotheses on these data in Subsection B).

Nonlinear elliptic problems with measure data have been studied in a number of papers. A quite efficient way to prove the existence of a solution to such problems is, as first shown in [3], to use an approximation method: taking a sequence of regular data which converges to the measure of the right-hand side, one can prove that the solutions corresponding to these regular data converge, in a sense, to a solution of the equation with measure data.

This method has been widely used to obtain different kinds of solutions to elliptic problems with measure data: see for example [4], [5], [2] or [7]. In each of these works, the first step to prove the convergence of the solutions corresponding to regular right-hand sides is, of course, to obtain estimates on these solutions in adequate spaces.

We intend here to obtain new estimates on elliptic problems with measure data.

One of the novelty of this paper is the presence of the convective term defined by $\Phi$; because of this term, the elliptic equation (1) is not coercive, and obtaining estimates on the solutions to this problem (with regular or singular right-hand side) is thus quite difficult. [10] give estimates for a noncoercive problem with right-hand side in $L^{1}(\Omega)$, using the tools of renormalized solutions. Here, we will rather use the method developed in [8] for linear noncoercive elliptic problems; this method was then adapted in [9] to nonlinear variational 
noncoercive elliptic problems and to finite volume schemes for linear noncoercive elliptic equations. We will see that, mixing the methods of [8] (to handle the noncoercive feature of the equation) and of [6] (to handle the singularity of the right-hand side), estimates on solutions to (1), similar to those already known when the problem is coercive, are easy to prove.

But the main originality of the work we present here is certainly the local estimate outside the support of the singular part of the right-hand side.

It is well-known that the solutions to elliptic problems with righthand side measures do not belong, in general, to the energy space $W_{0}^{1, p}(\Omega)$ associated to the equation (the global estimates we obtain on solutions to (1) are, roughly speaking, in $W_{0}^{1, q}(\Omega)$ spaces, with $q<N(p-1) /(N-1))$. This is quite obvious, since the right-hand side does not belong to the dual space of $W_{0}^{1, p}(\Omega)$. But if the singular part of the right-hand side is concentrated on some subset of $\Omega$, one can hope that, outside this subset, the solution is as regular as the operator allows.

We will indeed prove estimates (and thus regularity results), far from the support of $\mu$, on solutions to (1) in $W^{1, p}$. These estimates are not straightforward because, to study the solutions far from the support of $\mu$, we must introduce cut-off functions that not only have to satisfy some special properties but also entail the apparition of terms which are not easily bounded; we thus first use a bootstrap technique to reach estimates in $W^{1, q}$ for all $q<p$, and then, thanks to these estimates, we prove the desired bound in $W^{1, p}$.

In the rest of this section, we state the precise hypotheses on the data and the main results of this paper (global and local estimates, as well as their consequences on the regularity of solutions corresponding to right-hand side measures). In Section 2, we prove the global estimates on solutions to (1). Section 3, the biggest part of this paper, is devoted to the proof of the local estimates, far from the support of $\mu$. We then quickly show, in Section 4, how these estimates allow to obtain solutions with regularity properties when the right-hand side is a measure. Section 5 is an appendix with two easy technical results useful in the rest of the paper. 


\section{B. Hypotheses and notations}

$\Omega$ is a bounded open subset of $\mathbb{R}^{N}(N \geq 2) \cdot|\cdot|$ is the Euclidean norm in $\mathbb{R}^{N} ; B(e, r)$ and $\bar{B}(e, r)$ denote the open and closed ball in $\mathbb{R}^{N}$ of center $e$ and radius $r$. meas $(A)$ is the Lebesgue measure of a measurable set $A \subset \mathbb{R}^{N}$.

We take $p \in] 1, N]$; if $p<N$, we denote $N_{*}=N$ and, if $p=N$, we take $N_{*}>N$. We let $p^{*}=N_{*} p /\left(N_{*}-p\right) \cdot \bar{p}$ is a real number in $\left[N_{*} p /\left(N_{*}-p+1\right),\left(N_{*}-1\right) p /\left(N_{*}-p\right)\left[\left(^{*}\right)\right.\right.$.

$W_{0}^{1, r}(\Omega)$ denotes the usual Sobolev space, endowed with the norm $\|u\|_{W_{0}^{1, r}(\Omega)}=\||\nabla u|\|_{L^{r}(\Omega)} ; W^{-1, r^{\prime}}(\Omega)$ is the dual space of $W_{0}^{1, r}(\Omega)$. $\mathcal{M}(\Omega)$ is the space of bounded measures on $\Omega$, identified, through the Riesz theorem, to the dual space of $C_{c}(\Omega)$ (this last space is endowed with the supremum norm).

The hypotheses on the data of (1) are:

$a: \Omega \times \mathbb{R} \times \mathbb{R}^{N} \rightarrow \mathbb{R}^{N}$ is a Caratheodory function,

there exists $\nu>0$ and $\Theta \in L^{1}(\Omega)$ such that

$$
a(x, s, \xi) \cdot \xi \geq \nu|\xi|^{p}-\Theta(x)
$$

for a.e. $x \in \Omega$, for all $(s, \xi) \in \mathbb{R} \times \mathbb{R}^{N}$,

there exists $\beta>0$ and $h \in L^{p^{\prime}}(\Omega)$ such that

$$
|a(x, s, \xi)| \leq h(x)+\beta|s|^{\bar{p}-1}+\beta|\xi|^{p-1}
$$

for a.e. $x \in \Omega$, for all $(s, \xi) \in \mathbb{R} \times \mathbb{R}^{N}$,

$(a(x, s, \xi)-a(x, s, \eta)) \cdot(\xi-\eta)>0$ for a.e. $x \in \Omega$ and for all $(s, \xi, \eta) \in \mathbb{R} \times \mathbb{R}^{N} \times \mathbb{R}^{N}$ such that $\xi \neq \eta$.

\footnotetext{
${ }^{*}$ The choice $\bar{p} \geq N_{*} p /\left(N_{*}-p+1\right)$ is only made to avoid the introduction of a new notation in the following proofs; this restriction on $\bar{p}$ is not a problem: if $a$ satisfies (4) for $0 \leq \bar{p}<\left(N_{*}-1\right) p /\left(N_{*}-p\right)$, then it also satisfies this hypotheses for some $\bar{p} \in\left[N_{*} p /\left(N_{*}-p+1\right),\left(N_{*}-1\right) p /\left(N_{*}-p\right)\left[\right.\right.$ (notice that, indeed, $N_{*} p /\left(N_{*}-p+\right.$ 1) $\left.<\left(N_{*}-1\right) p /\left(N_{*}-p\right)\right)$.
} 
$\Phi: \Omega \times \mathbb{R} \rightarrow \mathbb{R}^{N}$ is a Caratheodory function such that there exists $g \in L^{N_{*} /(p-1)}(\Omega)$ satisfying

$|\Phi(x, s)| \leq g(x)\left(1+|s|^{p-1}\right)$ for a.e. $x \in \Omega$, for all $s \in \mathbb{R}$,

$$
\begin{gathered}
\mu \in \mathcal{M}(\Omega), f \in W^{-1, p^{\prime}}(\Omega), \\
\|\mu\|_{\mathcal{M}(\Omega)}+\|f\|_{W^{-1, p^{\prime}}(\Omega)} \leq \Lambda \text { and }
\end{gathered}
$$

$K$ is a compact set such that $\operatorname{supp}(\mu) \subset K$.

We know (see [9]) that, under Hypotheses (2) - (7), if $\mu$ belongs to $W^{-1, p^{\prime}}(\Omega)$, there exists at least one solution to (1) in the sense

$$
\left\{\begin{array}{l}
u \in W_{0}^{1, p}(\Omega) \\
\int_{\Omega} a(x, u, \nabla u) \cdot \nabla \varphi+\int_{\Omega} \Phi(x, u) \cdot \nabla \varphi \\
\quad=\langle\mu, \varphi\rangle_{W^{-1, p^{\prime}}(\Omega), W_{0}^{1, p}(\Omega)}+\langle f, \varphi\rangle_{W^{-1, p^{\prime}}(\Omega), W_{0}^{1, p}(\Omega)} \\
\quad \forall \varphi \in W_{0}^{1, p}(\Omega) .
\end{array}\right.
$$

\section{Main results}

Our main results are the following.

Theorem 1 (Global Estimates) Assume that Hypotheses (2)-(7) hold. If $\mu \in W^{-1, p^{\prime}}(\Omega)$ then, for all $\left.r \in\right] 0, N_{*}(p-1) /\left(N_{*}-p\right)[$ and all $s \in] 0, N_{*}(p-1) /\left(N_{*}-1\right)[$, there exists $C>0$ only depending on $(\Lambda, r, s)$ such that, for any solution $u$ of (8), we have

$$
\int_{\Omega}|u|^{r} \leq C \quad \text { and } \quad \int_{\Omega}|\nabla u|^{s} \leq C .
$$

Remark 1 Of course, this $C$ and all the constants in the sequel also depend on the other numerous data involved in the hypotheses, i.e. on $\left(\Omega, p, N_{*}, \nu, \Theta, g, h, \beta, \bar{p}\right)$, but we have chosen to emphasize the dependance only on the important data, that is to say the space in which we obtain the estimates (through the exponents $(r, s)$ in the global estimates), the norm of $\mu$ in $\mathcal{M}(\Omega)$ (through $\Lambda$ ) and, in the local estimates, the support of $\mu$ (through $K)$. In fact, a close 
examination of the proofs also shows that the constants appearing in the global estimates do not depend on $(h, \beta, \bar{p})$.

Remark 2 Notice that we can have $N_{*}(p-1) /\left(N_{*}-p\right) \leq 1$ and $N_{*}(p-1) /\left(N_{*}-1\right) \leq 1$, in which case Theorem 1 does not give estimates in Lebesgue or Sobolev spaces.

One can also notice that, once we have the result of Theorem 1, it is possible to obtain estimates on $u$ in the Marcinkiewicz space of exponent $N_{*}(p-1) /\left(N_{*}-p\right)$ and on $\nabla u$ in the Marcinkiewicz space of exponent $N_{*}(p-1) /\left(N_{*}-1\right)$ (but this does not improve the integrability properties of $u$ or $\nabla u)$.

Theorem 2 (Local Estimates) Under Hypotheses (2)-(7), if $\mu \in$ $W^{-1, p^{\prime}}(\Omega)$ then, for all $\varepsilon>0$, there exists $C>0$ only depending on $(\Lambda, K, \varepsilon)$ such that, for any solution $u$ of $(8)$, denoting $F=K+$ $\bar{B}(0, \varepsilon)$, we have

$$
\|u\|_{L^{p^{*}}(\Omega \backslash F)} \leq C \quad \text { and } \quad\|u\|_{W^{1, p}(\Omega \backslash F)} \leq C .
$$

As a consequence of these estimates, we obtain the following existence and regularity result on a nonlinear noncoercive elliptic equation with measure data.

Theorem 3 (Existence for a right-hand side measure) Assume that Hypotheses (2)-(7) hold. If $p>2-1 / N_{*}$, there exists a solution to (1) in the sense

$$
\left\{\begin{array}{c}
u \in \bigcap_{q<N_{*}(p-1) /\left(N_{*}-1\right)} W_{0}^{1, q}(\Omega), \\
\forall \varepsilon>0, \text { denoting } F_{\varepsilon}=\overline{\operatorname{supp}(\mu)+B(0, \varepsilon)} \\
u \in L^{p^{*}}\left(\Omega \backslash F_{\varepsilon}\right) \cap W^{1, p}\left(\Omega \backslash F_{\varepsilon}\right), \\
\int_{\Omega} a(x, u, \nabla u) \cdot \nabla \varphi+\int_{\Omega} \Phi(x, u) \cdot \nabla \varphi=\int_{\Omega} \varphi d \mu \\
\quad+\langle f, \varphi\rangle_{W^{-1, p^{\prime}}(\Omega), W_{0}^{1, p}(\Omega)}, \\
\forall \varphi \in \bigcup_{s>N_{*}} W_{0}^{1, s}(\Omega) .
\end{array}\right.
$$

Remark $3 \quad F_{\varepsilon}$ is defined as the closure of $\operatorname{supp}(\mu)+B(0, \varepsilon)$ to ensure that $W^{1, p}\left(\Omega \backslash F_{\varepsilon}\right)$ is a Sobolev space on an open set. 


\section{Global Estimates}

\section{A. Estimate on $\ln (1+|u|)$}

The following proposition is a nonlinear form of a proposition in [8].

Proposition 1 Under Hypotheses (2)-(7), if $\mu \in W^{-1, p^{\prime}}(\Omega)$ then there exists $C$ only depending on $\Lambda$ such that, for any solution $u$ of (8), we have $\|\ln (1+|u|)\|_{W_{0}^{1, p}(\Omega)} \leq C$.

\section{Proof of Proposition 1}

Let $\varphi(s)=\int_{0}^{s} 1 /(1+|t|)^{p} d t$. Using $\varphi(u)$ as a test function in (8), we find

$$
\begin{aligned}
& \int_{\Omega} a(x, u, \nabla u) \cdot \frac{\nabla u}{(1+|u|)^{p}} \\
& \leq \int_{\Omega} g\left(1+|u|^{p-1}\right) \frac{|\nabla u|}{(1+|u|)^{p}}+\langle\mu, \varphi(u)\rangle_{W^{-1, p^{\prime}}(\Omega), W_{0}^{1, p}(\Omega)} \\
& \quad+\langle f, \varphi(u)\rangle_{W^{-1, p^{\prime}}(\Omega), W_{0}^{1, p}(\Omega)} .
\end{aligned}
$$

But

$$
\begin{aligned}
a(x, u, \nabla u) \cdot \frac{\nabla u}{(1+|u|)^{p}} & \geq \nu \frac{|\nabla u|^{p}}{(1+|u|)^{p}}-\frac{\Theta(x)}{(1+|u|)^{p}} \\
& \geq \nu|\nabla(\ln (1+|u|))|^{p}-\Theta(x) .
\end{aligned}
$$

Since $1+|u|^{p-1} \leq 2(1+|u|)^{p-1}$, we can write

$$
\begin{aligned}
\int_{\Omega} g & \left(1+|u|^{p-1}\right) \frac{|\nabla u|}{(1+|u|)^{p}} \\
& \leq 2 \int_{\Omega} g \frac{|\nabla u|}{(1+|u|)} \\
& \leq 2|| g\left\|_{L^{p^{\prime}}(\Omega)}|||\nabla(\ln (1+|u|))|\right\|_{L^{p}(\Omega)}
\end{aligned}
$$

(notice that $g \in L^{p^{\prime}}(\Omega)$, since $p^{\prime} \leq N_{*} /(p-1)$ ).

Since $\left|\varphi^{\prime}(u)\right| \leq 1 /(1+|u|)$, we have

$$
|\nabla(\varphi(u))|=\left|\varphi^{\prime}(u)\right||\nabla u| \leq \frac{|\nabla u|}{1+|u|}=|\nabla(\ln (1+|u|))| .
$$

Thus, 


$$
\|\varphi(u)\|_{W_{0}^{1, p}(\Omega)}=\||\nabla(\varphi(u))|\|_{L^{p}(\Omega)} \leq\||\nabla(\ln (1+|u|))|\|_{L^{p}(\Omega)}
$$

and, $\varphi$ being bounded by $1 /(p-1)$, we get

$$
\begin{aligned}
& \left|\langle\mu, \varphi(u)\rangle_{W^{-1, p^{\prime}}(\Omega), W_{0}^{1, p}(\Omega)}+\langle f, \varphi(u)\rangle_{W^{-1, p^{\prime}}(\Omega), W_{0}^{1, p}(\Omega)}\right| \\
& \leq \frac{\Lambda}{p-1}+\Lambda|||\nabla(\ln (1+|u|))| \|_{L^{p}(\Omega)} .
\end{aligned}
$$

Injecting (11), (12) and (13) in (10), we obtain

$$
\nu\||\nabla(\ln (1+|u|))|\|_{L^{p}(\Omega)}^{p} \leq C+C|||\nabla(\ln (1+|u|))| \|_{L^{p}(\Omega)},
$$

with $C$ only depending on $\Lambda$, which concludes the proof (" $X^{p} \leq$ $C+C X "$ implies that $X$ is bounded, since $p>1$ ).

\section{B. Proof of the global estimates}

In the proof of the next proposition, we mix the ideas of [8] (or [9]) - to handle the noncoercive characteristic of the equation with the ideas of [6] — to handle the measure on the right-hand side of the equation.

Proposition 2 Let $\alpha>1$ and assume that Hypotheses (2)-(7) hold. If $\mu \in W^{-1, p^{\prime}}(\Omega)$ then there exists $C>0$ only depending on $(\Lambda, \alpha)$ such that, for any solution $u$ of $(8)$,

$$
\int_{\Omega} \frac{|\nabla u|^{p}}{(1+|u|)^{\alpha}} \leq C
$$

\section{Proof of Proposition 2}

We define

$$
\begin{aligned}
\varphi(s)= & \int_{0}^{s} \frac{d t}{(1+|t|)^{\alpha}}, T_{k}(s)=\max (-k, \min (s, k)) \\
\text { and } \quad & S_{k}(s)=s-T_{k}(s) .
\end{aligned}
$$

Step 1: estimate on $S_{k}(u)$.

Let $k>0$. Using $\varphi\left(S_{k}(u)\right)$ as a test function in (8), we get, since $\varphi$ is bounded by $1 /(\alpha-1)$,

$$
\int_{\Omega} a(x, u, \nabla u) \cdot \frac{\nabla\left(S_{k}(u)\right)}{\left(1+\left|S_{k}(u)\right|\right)^{\alpha}}
$$




$$
\begin{aligned}
\leq & \int_{\Omega} g\left(1+|u|^{p-1}\right) \frac{\left|\nabla\left(S_{k}(u)\right)\right|}{\left(1+\left|S_{k}(u)\right|\right)^{\alpha}}+\frac{\Lambda}{\alpha-1} \\
& +\Lambda\left\|\left|\nabla\left(\varphi\left(S_{k}(u)\right)\right)\right|\right\|_{L^{p}(\Omega)} .
\end{aligned}
$$

Since $\nabla\left(S_{k}(u)\right)=\mathbf{1}_{E_{k}} \nabla u$, where $E_{k}=\{|u|>k\}$ and $\mathbf{1}_{E_{k}}$ is the characteristic function of $E_{k}$, we have

$$
\begin{aligned}
a(x, u, \nabla u) \cdot \frac{\nabla\left(S_{k}(u)\right)}{\left(1+\left|S_{k}(u)\right|\right)^{\alpha}} & \geq \nu \frac{\left|\nabla\left(S_{k}(u)\right)\right|^{p}}{\left(1+\left|S_{k}(u)\right|\right)^{\alpha}}-\frac{\Theta}{\left(1+\left|S_{k}(u)\right|\right)^{\alpha}} \\
& \geq \nu \frac{\left|\nabla\left(S_{k}(u)\right)\right|^{p}}{\left(1+\left|S_{k}(u)\right|\right)^{\alpha}}-\Theta .
\end{aligned}
$$

$\left|\varphi^{\prime}(s)\right|^{p}=1 /(1+|s|)^{\alpha p} \leq 1 /(1+|s|)^{\alpha}$ for all $s \in \mathbb{R}$ (because $\alpha p \geq$ $\alpha)$, so that

$$
\left\|\left|\nabla\left(\varphi\left(S_{k}(u)\right)\right)\right|\right\|_{L^{p}(\Omega)} \leq\left(\int_{\Omega} \frac{\left|\nabla\left(S_{k}(u)\right)\right|^{p}}{\left(1+\left|S_{k}(u)\right|\right)^{\alpha}}\right)^{1 / p} .
$$

We have $|u| \leq k+\left|S_{k}(u)\right|$, which implies

$$
\begin{aligned}
& \int_{\Omega} g\left(1+|u|^{p-1}\right) \frac{\left|\nabla\left(S_{k}(u)\right)\right|}{\left(1+\left|S_{k}(u)\right|\right)^{\alpha}} \\
& \leq \int_{\Omega} g\left(1+2^{p-1} k^{p-1}+2^{p-1}\left|S_{k}(u)\right|^{p-1}\right) \frac{\left|\nabla\left(S_{k}(u)\right)\right|}{\left(1+\left|S_{k}(u)\right|\right)^{\alpha}} \\
& \leq\left.\left(1+2^{p-1} k^{p-1}\right)|| g\right|_{L^{p^{\prime}}(\Omega)}\left(\int_{\Omega} \frac{\left|\nabla\left(S_{k}(u)\right)\right|^{p}}{\left(1+\left|S_{k}(u)\right|\right)^{\alpha p}}\right)^{1 / p} \\
& \quad+2^{p-1} \int_{\Omega} g \frac{\left|S_{k}(u)\right|^{p-1}}{\left(1+\left|S_{k}(u)\right|\right)^{\frac{\alpha}{p^{\prime}}}} \frac{\left|\nabla\left(S_{k}(u)\right)\right|}{\left(1+\left|S_{k}(u)\right|\right)^{\frac{\alpha}{p}}} \\
& \leq C_{1}\left(1+k^{p-1}\right)\left(\int_{\Omega} \frac{\left|\nabla\left(S_{k}(u)\right)\right|^{p}}{\left(1+\left|S_{k}(u)\right|\right)^{\alpha}}\right)^{1 / p} \\
& \quad+C_{1}\left(\int_{\Omega} g^{p^{\prime}} \frac{\left|S_{k}(u)\right|^{p}}{\left(1+\left|S_{k}(u)\right|\right)^{\alpha}}\right)^{1 / p^{\prime}}\left(\int_{\Omega} \frac{\left|\nabla\left(S_{k}(u)\right)\right|^{p}}{\left(1+\left|S_{k}(u)\right|\right)^{\alpha}}\right)^{1 / p}
\end{aligned}
$$

(we have used $\left.\left(1+\left|S_{k}(u)\right|\right)^{\alpha p} \geq\left(1+\left|S_{k}(u)\right|\right)^{\alpha}\right)$.

Denoting

$$
A_{k}=\int_{\Omega} \frac{\left|\nabla\left(S_{k}(u)\right)\right|^{p}}{\left(1+\left|S_{k}(u)\right|\right)^{\alpha}} \quad \text { and } \quad \psi(s)=\frac{|s|}{(1+|s|)^{\frac{\alpha}{p}}},
$$


we have just proved that

$$
\begin{aligned}
& \int_{\Omega} g\left(1+|u|^{p-1}\right) \frac{\left|\nabla\left(S_{k}(u)\right)\right|}{\left(1+\left|S_{k}(u)\right|\right)^{\alpha}} \\
& \quad \leq C_{1}\left(1+k^{p-1}\right) A_{k}^{1 / p}+C_{1} A_{k}^{1 / p}\left(\int_{\Omega} g^{p^{\prime}} \psi\left(S_{k}(u)\right)^{p}\right)^{1 / p^{\prime}} .
\end{aligned}
$$

Let $\delta>0$ (fixed later on) and write $g=g_{1}+g_{2}$ with $g_{1} \in L^{\infty}(\Omega)$ and $g_{2} \in L^{N_{*} /(p-1)}(\Omega)$ such that $\left\|g_{2}\right\|_{L^{N_{*} /(p-1)}(\Omega)} \leq \delta$ (the choice of $\left(g_{1}, g_{2}\right)$ only depend on $\left.\delta\right)$. Thanks to Hölder's inequality with exponents $\left(N_{*} / p, N_{*} /\left(N_{*}-p\right)\right)$ and to the Sobolev injections, since $\psi\left(S_{k}(u)\right)=0$ outside $E_{k}$, we have

$$
\begin{aligned}
& \int_{\Omega} g^{p^{\prime}} \psi\left(S_{k}(u)\right)^{p} \\
& \leq\|g\|_{L^{\frac{N_{*}}{p-1}}\left(E_{k}\right)}^{p^{\prime}}\left\|\psi\left(S_{k}(u)\right)\right\|_{L^{\frac{N_{*} p}{N_{*}-p}}(\Omega)}^{p} \\
& \leq C_{2}\left(\left\|g_{1}\right\|_{L^{\frac{N_{*}}{p-1}}\left(E_{k}\right)}^{p^{p^{\prime}}}+\left\|g_{2}\right\|_{L^{\frac{N_{*}}{p^{-1}}\left(E_{k}\right)}}^{p^{p^{\prime}}}\right)\left\|\left|\nabla\left(\psi\left(S_{k}(u)\right)\right)\right|\right\|_{L^{p}(\Omega)}^{p} \\
& \leq C_{2}\left(\left\|g_{1}\right\|_{L^{\infty}(\Omega)}^{p^{\prime}(\Omega)} \operatorname{meas}\left(E_{k}\right)^{\frac{p}{N_{*}}}+\delta^{p^{\prime}}\right)\left\|\left|\nabla\left(\psi\left(S_{k}(u)\right)\right)\right|\right\|_{L^{p}(\Omega)}^{p} .
\end{aligned}
$$

Moreover, $\left|\psi^{\prime}(s)\right| \leq(1+\alpha / p) /(1+|s|)^{\alpha / p}$ for all $s \in \mathbb{R}$, so that

$$
\left|\nabla\left(\psi\left(S_{k}(u)\right)\right)\right|^{p} \leq\left(1+\frac{\alpha}{p}\right)^{p} \frac{\left|\nabla\left(S_{k}(u)\right)\right|^{p}}{\left(1+\left|S_{k}(u)\right|\right)^{\alpha}}
$$

and

$$
\int_{\Omega} g^{p^{\prime}} \psi\left(S_{k}(u)\right)^{p} \leq C_{3}\left(\left\|g_{1}\right\|_{L^{\infty}(\Omega)}^{p^{\prime}} \operatorname{meas}\left(E_{k}\right)^{\frac{p}{N_{*}}}+\delta^{p^{\prime}}\right) A_{k}
$$

with $C_{3}$ only depending on $\alpha$. Used in (17), this inequality allows us to write

$$
\begin{aligned}
\int_{\Omega} g\left(1+|u|^{p-1}\right) \frac{\left|\nabla\left(S_{k}(u)\right)\right|}{\left(1+\left|S_{k}(u)\right|\right)^{\alpha}} \\
\leq C_{4}\left(1+k^{p-1}\right) A_{k}^{1 / p} \\
\quad+C_{4}\left(\left\|g_{1}\right\|_{L^{\infty}(\Omega)}^{p^{\prime}} \operatorname{meas}\left(E_{k}\right)^{\frac{p}{N_{*}}}+\delta^{p^{\prime}}\right)^{1 / p^{\prime}} A_{k}
\end{aligned}
$$

where $C_{4}$ only depends on $\alpha$. 
(14), (15), (16) and (18) give

$$
\begin{aligned}
A_{k} \leq & C_{5}+C_{5}\left(1+k^{p-1}\right) A_{k}^{1 / p} \\
& +C_{5}\left(\left\|g_{1}\right\|_{L^{\infty}(\Omega)}^{p^{\prime}} \operatorname{meas}\left(E_{k}\right)^{\frac{p}{N_{*}}}+\delta^{p^{\prime}}\right)^{1 / p^{\prime}} A_{k} \\
\leq & C_{5}+C_{5}\left(1+k^{p-1}\right) A_{k}^{1 / p} \\
& +C_{5}\left(\left\|g_{1}\right\|_{L^{\infty}(\Omega)} \operatorname{meas}\left(E_{k}\right)^{\frac{p-1}{N_{*}}}+\delta\right) A_{k}
\end{aligned}
$$

where $C_{5}$ only depends on $(\Lambda, \alpha)$ (we have used the fact that, for $\left.(s, t) \in \mathbb{R}^{+},(s+t)^{1 / p^{\prime}} \leq s^{1 / p^{\prime}}+t^{1 / p^{\prime}}\right)$.

Set $\delta=1 /\left(4 C_{5}\right)$, which only depends on $(\Lambda, \alpha)$. By Proposition 1 , Tchebychev's inequality and Poincaré's inequality, we have

$$
\operatorname{meas}\left(E_{k}\right) \leq \frac{\|\ln (1+|u|)\|_{L^{p}(\Omega)}^{p}}{(\ln (1+k))^{p}} \leq \frac{C_{6}}{(\ln (1+k))^{p}}
$$

with $C_{6}$ only depending on $\Lambda$. There exists thus $k_{0}$ only depending on $(\Lambda, \alpha)$, such that $C_{5}\left\|g_{1}\right\|_{L^{\infty}(\Omega)} \operatorname{meas}\left(E_{k_{0}}\right)^{(p-1) / N_{*}} \leq 1 / 4$.

With these choices of $\delta$ and $k_{0}$, (19) becomes $A_{k_{0}} \leq C_{5}+C_{5}(1+$ $\left.k_{0}^{p-1}\right) A_{k_{0}}^{1 / p}+A_{k_{0}} / 2$, which leads to $A_{k_{0}} \leq 2 C_{5}+2 C_{5}\left(1+k_{0}^{p-1}\right) A_{k_{0}}^{1 / p}$. By Young's inequality, we obtain thus $C_{7}$ only depending on $(\Lambda, \alpha)$, such that $A_{k_{0}} \leq C_{7}$, that is to say

$$
\int_{\Omega} \frac{\left|\nabla\left(S_{k_{0}}(u)\right)\right|^{p}}{\left(1+\left|S_{k_{0}}(u)\right|\right)^{\alpha}} \leq C_{7}
$$

Step 2: conclusion.

By Proposition 1, we have $\|\left.\ln (1+|u|)\right|_{W_{0}^{1, p}(\Omega)} ^{p} \leq C_{8}$ with $C_{8}$ only depending on $\Lambda$, thus

$$
\begin{aligned}
\int_{\Omega}\left|\nabla\left(T_{k_{0}}(u)\right)\right|^{p} & =\int_{\left\{|u| \leq k_{0}\right\}}|\nabla u|^{p} \\
& \leq\left(1+k_{0}\right)^{p} \int_{\left\{|u| \leq k_{0}\right\}} \frac{|\nabla u|^{p}}{(1+|u|)^{p}} \\
& \leq\left(1+k_{0}\right)^{p} C_{8} .
\end{aligned}
$$


Since $u=T_{k_{0}}(u)+S_{k_{0}}(u)$ and $1+\left|S_{k_{0}}(u)\right| \leq 1+|u|$, we have, by (20) and (21),

$$
\begin{aligned}
\int_{\Omega} \frac{|\nabla u|^{p}}{(1+|u|)^{\alpha}} & \leq 2^{p} \int_{\Omega} \frac{\left|\nabla\left(T_{k_{0}}(u)\right)\right|^{p}}{(1+|u|)^{\alpha}}+2^{p} \int_{\Omega} \frac{\left|\nabla\left(S_{k_{0}}(u)\right)\right|^{p}}{(1+|u|)^{\alpha}} \\
& \leq 2^{p} \int_{\Omega}\left|\nabla\left(T_{k_{0}}(u)\right)\right|^{p}+2^{p} \int_{\Omega} \frac{\left|\nabla\left(S_{k_{0}}(u)\right)\right|^{p}}{\left(1+\left|S_{k_{0}}(u)\right|\right)^{\alpha}} \\
& \leq 2^{p}\left(1+k_{0}\right)^{p} C_{8}+2^{p} C_{7},
\end{aligned}
$$

which concludes the proof.

We can now prove the global estimates theorem.

\section{Proof of Theorem 1}

Since $r<N_{*}(p-1) /\left(N_{*}-p\right)$ and $s<N_{*}(p-1) /\left(N_{*}-1\right)$, there exists $\alpha \in] 1, p[$ only depending on $(r, s)$ such that

$$
r<\frac{N_{*}(p-\alpha)}{N_{*}-p} \quad \text { and } \quad s<\frac{N_{*}(p-\alpha)}{N_{*}-\alpha} .
$$

Let $C_{1}$ only depending on $(\Lambda, \alpha)$ (i.e. on $\left.(\Lambda, r, s)\right)$ given by Proposition 2 . We have, for all $k \geq 1$,

$$
\begin{aligned}
\int_{\Omega}\left|\nabla\left(T_{k}(u)\right)\right|^{p} & =\int_{\{|u| \leq k\}}|\nabla u|^{p} \\
& \leq(1+k)^{\alpha} \int_{\Omega} \frac{|\nabla u|^{p}}{(1+|u|)^{\alpha}} \\
& \leq C_{1}(1+k)^{\alpha} \\
& \leq 2^{\alpha} C_{1} k^{\alpha} .
\end{aligned}
$$

Since $0<r<N_{*}(p-\alpha) /\left(N_{*}-p\right)$ and $0<s<N_{*}(p-\alpha) /\left(N_{*}-\right.$ $\alpha)$, the proof is then an easy consequence of Lemmas 2 and 1.

\section{Local Estimates}

\section{A. Preliminary results}

Proposition 3 Suppose that Hypotheses (2)—(7) hold and that $\mu \in W^{-1, p^{\prime}}(\Omega)$. Let $\theta \in C^{\infty}\left(\mathbb{R}^{N} ; \mathbb{R}^{+}\right)$be such that 


$$
\forall m>0, \theta^{m} \in C^{\infty}\left(\mathbb{R}^{N} ; \mathbb{R}^{+}\right),
$$

$\forall m \in] 0,1\left[, \exists Q_{m}\right.$ such that $|\nabla \theta| \leq Q_{m} \theta^{m}$, $\theta=0$ on a neighborhood of $K$.

Then there exists $C$ only depending on $(\Lambda, \theta)$ such that, if $u$ is a solution to (8), we have, for all $\alpha \in] 0,1]$ and all $k \geq 0$,

$$
\begin{aligned}
\int_{\Omega}\left|\nabla\left(T_{k}(\theta u)\right)\right|^{p} \leq & C\left(1+k^{\alpha}\right) \\
& +C k^{\alpha} \int_{\Omega \cap \operatorname{supp}(\theta)}\left(|u|^{\bar{p}-\alpha}+|\nabla u|^{p-\alpha}\right) .
\end{aligned}
$$

Remark 4 Functions satisfying (23) exist and will be constructed in the proof of Proposition 4.

\section{Proof of Proposition 3}

$\theta$ and $\theta^{p-1}$ being regular functions, we can take $\theta^{p-1} T_{k}(\theta u)$ as a test function in (8); this gives, since $\theta=0$ on a neighborhood of $\operatorname{supp}(\mu)$,

$$
\begin{aligned}
& \left\langle f, \theta^{p-1} T_{k}(\theta u)\right\rangle_{W^{-1, p^{\prime}}(\Omega), W_{0}^{1, p}(\Omega)} \\
& =\int_{\Omega} a(x, u, \nabla u) \cdot \nabla\left(\theta^{p-1} T_{k}(\theta u)\right)+\int_{\Omega} \Phi(x, u) \cdot \nabla\left(\theta^{p-1} T_{k}(\theta u)\right) \\
& =\int_{\Omega} \theta^{p-1} a(x, u, \nabla u) \cdot \nabla\left(T_{k}(\theta u)\right) \\
& \quad+\int_{\Omega} T_{k}(\theta u) a(x, u, \nabla u) \cdot \nabla\left(\theta^{p-1}\right) \\
& \quad+\int_{\Omega} \theta^{p-1} \Phi(x, u) \cdot \nabla\left(T_{k}(\theta u)\right)+\int_{\Omega} T_{k}(\theta u) \Phi(x, u) \cdot \nabla\left(\theta^{p-1}\right) \\
& =\int_{\Omega} \theta^{p} \mathbf{1}_{\{|\theta u| \leq k\}} a(x, u, \nabla u) \cdot \nabla u \\
& \quad+\int_{\Omega} \theta^{p-1} u \mathbf{1}_{\{|\theta u| \leq k\}} a(x, u, \nabla u) \cdot \nabla \theta \\
& \quad+\int_{\Omega} T_{k}(\theta u) a(x, u, \nabla u) \cdot \nabla\left(\theta^{p-1}\right) \\
& \quad+\int_{\Omega} \mathbf{1}_{\{|\theta u| \leq k\}} \theta^{p} \Phi(x, u) \cdot \nabla u+\int_{\Omega} \mathbf{1}_{\{|\theta u| \leq k\}} \theta^{p-1} u \Phi(x, u) \cdot \nabla \theta
\end{aligned}
$$




$$
+\int_{\Omega} T_{k}(\theta u) \Phi(x, u) \cdot \nabla\left(\theta^{p-1}\right) .
$$

We have $\theta^{p-1}|\nabla \theta| \leq C_{1} \theta$ on $\Omega$, with $C_{1}$ only depending on $\theta$ (indeed, if $p \geq 2$, we just use the fact that $\theta^{p-2}$ and $\nabla \theta$ are bounded on $\Omega$ and, if $p \in] 1,2\left[\right.$, we use (23) to get $|\nabla \theta| \leq Q_{2-p} \theta^{2-p}$ ); moreover, $\nabla\left(\theta^{p-1}\right)$ is bounded on $\Omega$ (say by $C_{2}$ ). Thus, thanks to Hypotheses (3) and (4), we have

$$
\begin{aligned}
& \int_{\Omega} \theta^{p} \mathbf{1}_{\{|\theta u| \leq k\}} a(x, u, \nabla u) \cdot \nabla u \\
&+\int_{\Omega} \theta^{p-1} u \mathbf{1}_{\{|\theta u| \leq k\}} a(x, u, \nabla u) \cdot \nabla \theta \\
&+\int_{\Omega} T_{k}(\theta u) a(x, u, \nabla u) \cdot \nabla\left(\theta^{p-1}\right) \\
& \geq \nu \int_{\Omega} \mathbf{1}_{\{|\theta u| \leq k\}} \theta^{p}|\nabla u|^{p}-\int_{\Omega} \theta^{p} \Theta \\
&-\int_{\Omega} \mathbf{1}_{\{|\theta u| \leq k\}}\left(h+\beta|u|^{\bar{p}-1}+\beta|\nabla u|^{p-1}\right) \theta^{p-1}|\nabla \theta||u| \\
&-\int_{\Omega}\left(h+\beta|u|^{\bar{p}-1}+\beta|\nabla u|^{p-1}\right)\left|\nabla\left(\theta^{p-1}\right)\right|\left|T_{k}(\theta u)\right| \\
& \geq \nu \int_{\Omega} \mathbf{1}_{\{|\theta u| \leq k\}} \theta^{p}|\nabla u|^{p}-\int_{\Omega} \theta^{p} \Theta \\
&-C_{1} \int_{\Omega} \mathbf{1}_{\{|\theta u| \leq k\}}\left(h+\beta|u|^{\bar{p}-1}+\beta|\nabla u|^{p-1}\right)|\theta u| \\
&-C_{2} \int_{\Omega}\left(h+\beta|u|^{\bar{p}-1}+\beta|\nabla u|^{p-1}\right)\left|T_{k}(\theta u)\right| \\
& \geq \nu \int_{\Omega} \mathbf{1}_{\{|\theta u| \leq k\}} \theta^{p}|\nabla u|^{p}-\int_{\Omega} \theta^{p} \Theta \\
&-\left(C_{1}+C_{2}\right) \int_{\Omega}\left(h+\beta|u|^{\bar{p}-1}+\beta|\nabla u|^{p-1}\right)\left|T_{k}(\theta u)\right| . \quad(25)
\end{aligned}
$$

By Hypothesis (6), we also have, with the same $\left(C_{1}, C_{2}\right)$ as before and using the fact that $s t \leq s^{r}+t^{r^{\prime}}$ if $1<r<\infty$ and $(s, t) \in \mathbb{R}^{+}$ (simplified Young's inequality),

$$
\int_{\Omega} \mathbf{1}_{\{|\theta u| \leq k\}} \theta^{p} \Phi(x, u) \cdot \nabla u+\int_{\Omega} \mathbf{1}_{\{|\theta u| \leq k\}} \theta^{p-1} u \Phi(x, u) \cdot \nabla \theta
$$




$$
\begin{aligned}
+\int_{\Omega} T_{k}( & \theta u) \Phi(x, u) \cdot \nabla\left(\theta^{p-1}\right) \\
\geq & -\int_{\Omega} \mathbf{1}_{\{|\theta u| \leq k\}} \theta^{p} g\left(1+|u|^{p-1}\right)|\nabla u| \\
& -\int_{\Omega} \mathbf{1}_{\{|\theta u| \leq k\}} \theta^{p-1}|\nabla \theta||u| g\left(1+|u|^{p-1}\right) \\
& -C_{2} \int_{\Omega} g\left(1+|u|^{p-1}\right)\left|T_{k}(\theta u)\right| \\
\geq & -\int_{\Omega} \mathbf{1}_{\{|\theta u| \leq k\}}|\theta \nabla u| \theta^{p-1} g\left(1+|u|^{p-1}\right) \\
& -C_{1} \int_{\Omega} \mathbf{1}_{\{|\theta u| \leq k\}}|\theta u| g\left(1+|u|^{p-1}\right) \\
& -C_{2} \int_{\Omega} g\left(1+|u|^{p-1}\right)\left|T_{k}(\theta u)\right| \\
\geq & -\frac{\nu}{2} \int_{\Omega} \mathbf{1}_{\{|\theta u| \leq k\}}|\theta \nabla u|^{p} \\
& -\left(\frac{2}{\nu}\right)^{p^{\prime} / p} \int_{\Omega} \mathbf{1}_{\{|\theta u| \leq k\}} \theta^{p} g^{p^{\prime}}\left(1+|u|^{p-1}\right)^{p^{\prime}} \\
& -\left(C_{1}+C_{2}\right) \int_{\Omega}\left|T_{k}(\theta u)\right| g\left(1+|u|^{p-1}\right) \\
\geq & -\frac{\nu}{2} \int_{\Omega} \mathbf{1}_{\{|\theta u| \leq k\}} \theta^{p}|\nabla u|^{p} \\
& -2^{p^{\prime}}\left(\frac{2}{\nu}\right)^{p^{\prime} / p} \int_{\Omega} \mathbf{1}_{\{|\theta u| \leq k\}} \theta^{p} g^{p^{\prime}}\left(1+|u|^{p}\right) \\
& -\left(C_{1}+C_{2}\right) \int_{\Omega}\left|T_{k}(\theta u)\right| g\left(1+|u|^{p-1}\right) . \\
&
\end{aligned}
$$

Gathering (24), (25) and (26), we obtain $C_{3}$ only depending on $\theta$ such that

$$
\begin{aligned}
& \left\langle f, \theta^{p-1} T_{k}(\theta u)\right\rangle_{W^{-1, p^{\prime}}(\Omega), W_{0}^{1, p}(\Omega)} \\
& \geq \frac{\nu}{2} \int_{\{|\theta u| \leq k\}} \theta^{p}|\nabla u|^{p} \\
& \quad-C_{3} \int_{\Omega}\left(h+|u|^{\bar{p}-1}+|\nabla u|^{p-1}+g+g|u|^{p-1}\right)\left|T_{k}(\theta u)\right|
\end{aligned}
$$




$$
-C_{3} \int_{\{|\theta u| \leq k\}}\left(\theta^{p} g^{p^{\prime}}+|\theta u|^{p} g^{p^{\prime}}\right)-C_{3} .
$$

This inequality allows us to write

$$
\begin{aligned}
& \int_{\Omega}\left|\nabla\left(T_{k}(\theta u)\right)\right|^{p} \\
& =\int_{\{|\theta u| \leq k\}}|\nabla(\theta u)|^{p} \\
& \leq 2^{p} \int_{\{|\theta u| \leq k\}}|\nabla \theta|^{p}|u|^{p}+2^{p} \int_{\{|\theta u| \leq k\}} \theta^{p}|\nabla u|^{p} \\
& \leq C_{4} \int_{\{|\theta u| \leq k\}}|\nabla \theta|^{p}|u|^{p}+C_{4}\left\langle f, \theta^{p-1} T_{k}(\theta u)\right\rangle_{W^{-1, p^{\prime}}(\Omega), W_{0}^{1, p}(\Omega)} \\
& \quad+C_{4} \int_{\Omega}\left(h+g+g|u|^{p-1}+|u|^{\bar{p}-1}+|\nabla u|^{p-1}\right)\left|T_{k}(\theta u)\right| \\
& \quad+C_{4} \int_{\Omega} g^{p^{\prime}}\left|T_{k}(\theta u)\right|^{p}+C_{4},
\end{aligned}
$$

where $C_{4}$ only depends on $\theta$ (notice that $\theta^{p} g^{p^{\prime}} \in L^{1}(\Omega)$ since $p^{\prime} \leq$ $N_{*} /(p-1)$ and $\theta$ is bounded on $\left.\Omega\right)$.

Take $\delta>0$ (to be fixed later on); there exists $g_{1} \in L^{\infty}(\Omega)$ and $g_{2} \in L^{N_{*} /(p-1)}(\Omega)$, only depending on $\delta$, such that $g=g_{1}+g_{2}$ and $\left\|g_{2}\right\|_{L^{N_{*} /(p-1)}(\Omega)} \leq \delta$.

We have $|\nabla \theta|^{p} \leq C_{5} \theta$ on $\Omega$ (this is (23) with $m=1 / p$ ), where $C_{5}$ only depends on $\theta$. Thus, (27) gives $C_{6}$ only depending on $\theta$ such that

$$
\begin{aligned}
& \int_{\Omega}\left|\nabla\left(T_{k}(\theta u)\right)\right|^{p} \\
& \leq C_{6} \int_{\{|\theta u| \leq k\}}|\theta u||u|^{p-1}+C_{6}\left\langle f, \theta^{p-1} T_{k}(\theta u)\right\rangle_{W^{-1, p^{\prime}}(\Omega), W_{0}^{1, p}(\Omega)} \\
& \quad+C_{6} \int_{\Omega \cap \operatorname{supp}(\theta)}\left(h+g+g|u|^{p-1}+|u|^{\bar{p}-1}+|\nabla u|^{p-1}\right)\left|T_{k}(\theta u)\right| \\
& \quad+C_{6}|| g_{1} \|_{L^{\infty}(\Omega)}^{p^{\prime}} \int_{\Omega}\left|T_{k}(\theta u)\right||\theta u|^{p-1}+C_{6} \int_{\Omega} g_{2}^{p^{\prime}}\left|T_{k}(\theta u)\right|^{p}+C_{6} \\
& \leq C_{6}\left\langle f, \theta^{p-1} T_{k}(\theta u)\right\rangle_{W^{-1, p^{\prime}}(\Omega), W_{0}^{1, p}(\Omega)}
\end{aligned}
$$




$$
\begin{aligned}
& +C_{6} \int_{\Omega \cap \operatorname{supp}(\theta)}\left(h+g+(1+g)|u|^{p-1}\right. \\
& \left.+|u|^{\bar{p}-1}+|\nabla u|^{p-1}\right)\left|T_{k}(\theta u)\right| \\
& +C_{6}|| g_{1}||_{L^{\infty}(\Omega)}^{p^{\prime}}|| \theta||_{L^{\infty}(\Omega)}^{p-1} \int_{\Omega}|u|^{p-1}\left|T_{k}(\theta u)\right| \\
& +C_{6} \int_{\Omega} g_{2}^{p^{\prime}}\left|T_{k}(\theta u)\right|^{p}+C_{6} .
\end{aligned}
$$

But, $\theta^{p-1}$ being regular, by the Hölder inequality, the Sobolev injection and the Poincaré inequality, we have

$$
\begin{aligned}
& C_{6}\left\langle f, \theta^{p-1} T_{k}(\theta u)\right\rangle_{W^{-1, p^{\prime}}(\Omega), W_{0}^{1, p}(\Omega)} \\
& +C_{6} \int_{\Omega \cap \operatorname{supp}(\theta)}(h+g)\left|T_{k}(\theta u)\right|+C_{6} \int_{\Omega} g_{2}^{p^{\prime}}\left|T_{k}(\theta u)\right|^{p} \\
& \leq C_{6} \Lambda\left\|\theta^{p-1} T_{k}(\theta u)\right\|_{W_{0}^{1, p}(\Omega)} \\
& +C_{6}\|h+g\|_{L^{p^{\prime}}(\Omega)}\left\|T_{k}(\theta u)\right\|_{L^{p}(\Omega)} \\
& +C_{6}\left\|\left.g_{2}\right|_{L^{\frac{N_{*}}{p-1}}(\Omega)} ^{\frac{p}{p-1}}\right\| T_{k}(\theta u) \|_{L^{\frac{N_{*} p}{N_{*}-p}}(\Omega)}^{p} \\
& \leq\left(C_{7} \Lambda+C_{7}|| h+g \|_{L^{p^{\prime}}(\Omega)}\right)||\left|\nabla\left(T_{k}(\theta u)\right)\right| \|_{L^{p}(\Omega)} \\
& +C_{7} \delta^{p^{\prime}}\left\|\left|\nabla\left(T_{k}(\theta u)\right)\right|\right\|_{L^{p}(\Omega)}^{p} \\
& \leq \frac{1}{p^{\prime}}\left(C_{7} \Lambda+C_{7}\|h+g\|_{L^{p^{\prime}}(\Omega)}\right)^{p^{\prime}} \\
& +\left(\frac{1}{p}+C_{7} \delta^{p^{\prime}}\right)\left\|\left|\nabla\left(T_{k}(\theta u)\right)\right|\right\|_{L^{p}(\Omega)}^{p},
\end{aligned}
$$

where $C_{7}$ only depends on $\theta$.

Fix now $\delta>0$ such that $1 / p+C_{7} \delta^{p^{\prime}}<1$ (such a choice of $\delta$ only depends on $\theta$ ). Returning to (28), we find $C_{8}$ only depending on $(\Lambda, \theta)$ such that

$$
\begin{aligned}
& \int_{\Omega}\left|\nabla\left(T_{k}(\theta u)\right)\right|^{p} \leq C_{8} \\
& \quad+C_{8} \int_{\Omega \cap \operatorname{supp}(\theta)}\left((1+g)|u|^{p-1}+|u|^{\bar{p}-1}+|\nabla u|^{p-1}\right)\left|T_{k}(\theta u)\right| \cdot(29
\end{aligned}
$$


Let $\alpha \in] 0,1]$. We have, if $\alpha<1$,

$$
\begin{aligned}
\left|T_{k}(\theta u)\right| & =\left|T_{k}(\theta u)\right|^{\alpha}\left|T_{k}(\theta u)\right|^{1-\alpha} \\
& \leq k^{\alpha} \theta^{1-\alpha}|u|^{1-\alpha} \\
& \leq\left(1+\|\left.\theta\right|_{L^{\infty}(\Omega)}\right) k^{\alpha}|u|^{1-\alpha},
\end{aligned}
$$

so that

$$
\begin{aligned}
\left(|u|^{\bar{p}-1}\right. & \left.+|\nabla u|^{p-1}\right)\left|T_{k}(\theta u)\right| \\
& \leq\left(1+\left.|| \theta\right|_{L^{\infty}(\Omega)}\right) k^{\alpha}\left(|u|^{\bar{p}-\alpha}+|\nabla u|^{p-1}|u|^{1-\alpha}\right) .
\end{aligned}
$$

Using the simplified Young's inequality with $(p-\alpha) /(p-1)>1$, we find

$$
|\nabla u|^{p-1}|u|^{1-\alpha} \leq|\nabla u|^{p-\alpha}+|u|^{p-\alpha},
$$

which gives

$$
\begin{aligned}
\left(|u|^{\bar{p}-1}\right. & \left.+|\nabla u|^{p-1}\right)\left|T_{k}(\theta u)\right| \\
& \leq\left(1+\left.|| \theta\right|_{L^{\infty}(\Omega)}\right) k^{\alpha}\left(|u|^{\bar{p}-\alpha}+|u|^{p-\alpha}+|\nabla u|^{p-\alpha}\right) .
\end{aligned}
$$

Notice that $N_{*} /\left(N_{*}-p+1\right)>1$ (because $p>1$ ), so that $\bar{p}>p$. In particular, $|u|^{p-\alpha} \leq 1+|u|^{\bar{p}-\alpha}$ and

$$
\begin{aligned}
\left(|u|^{\bar{p}-1}\right. & \left.+|\nabla u|^{p-1}\right)\left|T_{k}(\theta u)\right| \\
& \leq\left(1+\left.|| \theta\right|_{L^{\infty}(\Omega)}\right) k^{\alpha}\left(1+2|u|^{\bar{p}-\alpha}+|\nabla u|^{p-\alpha}\right) .
\end{aligned}
$$

This inequality is still valid if $\alpha=1$ (we simply bound $T_{k}(\theta u)$ by $k$ ).

Thanks again to (30) if $\alpha<1$, or bounding $T_{k}(\theta u)$ by $k$ if $\alpha=1$, we can also write

$$
\begin{aligned}
& (1+g)|u|^{p-1}\left|T_{k}(\theta u)\right| \\
& \quad \leq\left(1+\|\theta\|_{L^{\infty}(\Omega)}\right) k^{\alpha}(1+g)|u|^{p-\alpha}
\end{aligned}
$$

which gives, by the simplified Young's inequality with $(\bar{p}-\alpha) /(p-$ $\alpha)>1$,

$$
\begin{aligned}
& (1+g)|u|^{p-1}\left|T_{k}(\theta u)\right| \\
& \quad \leq\left(1+\|\theta\|_{L^{\infty}(\Omega)}\right) k^{\alpha}\left((1+g)^{\frac{\bar{p}-\alpha}{\bar{p}-p}}+|u|^{\bar{p}-\alpha}\right) .
\end{aligned}
$$

We have $\bar{p} \geq N_{*} p /\left(N_{*}-p+1\right)$, which implies 


$$
\frac{\bar{p}-\alpha}{\bar{p}-p} \leq \frac{\bar{p}}{\bar{p}-p}=\frac{1}{1-p / \bar{p}} \leq \frac{1}{1-\left(N_{*}-p+1\right) / N_{*}}=\frac{N_{*}}{p-1}
$$

and $(1+g)^{(\bar{p}-\alpha) /(\bar{p}-p)} \leq(1+g)^{N_{*} /(p-1)} \in L^{1}(\Omega)$. Using this last inequality in (32) and injecting the result in (29) along with (31), we obtain

$$
\begin{aligned}
& \int_{\Omega}\left|\nabla\left(T_{k}(\theta u)\right)\right|^{p} \\
& \quad \leq C_{8}+C_{9} k^{\alpha}+C_{9} k^{\alpha} \int_{\Omega \cap \operatorname{supp}(\theta)}\left(|u|^{\bar{p}-\alpha}+|\nabla u|^{p-\alpha}\right),
\end{aligned}
$$

with $C_{9}$ only depending on $(\Lambda, \theta)$, which concludes the proof.

Corollary 1 Let $M \geq 0$ and $\alpha \in] 0,1]$. Under Hypotheses (2)-(7), if $\mu \in W^{-1, p^{\prime}}(\Omega)$, $\theta$ satisfies (23) and $u$ is a solution to (8) which satisfies

$$
\int_{\Omega \cap \operatorname{supp}(\theta)}\left(|u|^{\bar{p}-\alpha}+|\nabla u|^{p-\alpha}\right) \leq M
$$

then, for all $0<r<N_{*}(p-\alpha) /\left(N_{*}-p\right)$ and all $0<s<N_{*}(p-$ $\alpha) /\left(N_{*}-\alpha\right)$, there exists $C$ only depending on $(\Lambda, \theta, M, \alpha, r, s)$ such that

$$
\int_{\Omega}\left(|\theta u|^{r}+|\nabla(\theta u)|^{s}\right) \leq C
$$

Proof of Corollary 1.

By Proposition 3, we have $C_{1}$ only depending on $(\Lambda, \theta)$ such that, for all $k \geq 1$,

$$
\int_{\Omega}\left|\nabla\left(T_{k}(\theta u)\right)\right|^{p} \leq C_{1}+C_{1} k^{\alpha}+C_{1} M k^{\alpha} \leq\left(2 C_{1}+C_{1} M\right) k^{\alpha} .
$$

The corollary is then an easy consequence of Lemmas 2 and 1.

\section{B. Proof of the local estimates}

We first prove, thanks to a bootstrap technique based on Corollary 1 , local estimates in $W^{1, q}$ for all $q<p$ and then, using these estimates 
for $q$ close enough to $p$, we deduce Theorem 2 .

Proposition 4 Assume that Hypotheses (2)-(7) hold. Let $\varepsilon>0$ and denote $F=K+\bar{B}(0, \varepsilon)$; if $1 \leq q<p$, then there exists $C$ only depending on $(\Lambda, K, \varepsilon, q)$ such that, for all $u$ solution to (8),

$$
\|u\|_{L^{\frac{N_{*}-q}{N_{*}-q}}(\Omega \backslash F)}+\|u\|_{W^{1, q}(\Omega \backslash F)} \leq C .
$$

\section{Proof of Proposition 4}

The proof is based on an induction reasoning, which uses the following sequence: $\alpha_{1}=1$ and, for $n \geq 2$,

$$
\alpha_{n}=\max \left(\bar{p}-\frac{N_{*}\left(p-\alpha_{n-1}\right)}{N_{*}-p} ; \frac{\left(N_{*}-p\right) \alpha_{n-1}}{N_{*}-\alpha_{n-1}}\right) .
$$

Step 1: study of $\left(\alpha_{n}\right)_{n \geq 1}$.

Let us prove by induction that $\left(\alpha_{n}\right)_{n \geq 1}$ is a decreasing sequence of numbers in ]0,1]. Indeed, suppose that, for $\left.\left.n \geq 2, \alpha_{n-1} \in\right] 0,1\right]$; then $\left.\left(N_{*}-p\right) /\left(N_{*}-\alpha_{n-1}\right) \in\right] 0,1\left[\right.$ (because $\left.p>1 \geq \alpha_{n-1}\right)$, so that $\left.\left(N_{*}-p\right) \alpha_{n-1} /\left(N_{*}-\alpha_{n-1}\right) \in\right] 0, \alpha_{n-1}[$; moreover, by definition of $\bar{p}$,

$$
\begin{aligned}
\bar{p}-\frac{N_{*}\left(p-\alpha_{n-1}\right)}{N_{*}-p} & <\frac{\left(N_{*}-1\right) p}{N_{*}-p}-\frac{N_{*}\left(p-\alpha_{n-1}\right)}{N_{*}-p} \\
& =\frac{N_{*} \alpha_{n-1}-p}{N_{*}-p} \\
& =\alpha_{n-1}-\left(1-\alpha_{n-1}\right) \frac{p}{N_{*}-p},
\end{aligned}
$$

and this last quantity belongs to ] $-\infty, \alpha_{n-1}$ ] (because $1-\alpha_{n-1} \geq$ $0)$; thus, $\alpha_{n}$ belongs to $] 0, \alpha_{n-1}[$.

Denote $\alpha_{\infty} \in\left[0, \alpha_{1}\right.$ [ the limit of the decreasing sequence $\left(\alpha_{n}\right)_{n \geq 1}$. By passing to the limit $n \rightarrow \infty$ in (33) (the right-hand side of this equality is a continuous function of $\alpha_{n-1}$ on $\left.[0,1]\right)$, we obtain

$$
\alpha_{\infty}=\max \left(\bar{p}-\frac{N_{*}\left(p-\alpha_{\infty}\right)}{N_{*}-p} ; \frac{\left(N_{*}-p\right) \alpha_{\infty}}{N_{*}-\alpha_{\infty}}\right) .
$$

This maximum cannot be $\bar{p}-N_{*}\left(p-\alpha_{\infty}\right) /\left(N_{*}-p\right)$, because this would lead (thanks to the computations of (34) applied to $\alpha_{\infty}$ instead of $\left.\alpha_{n-1}\right)$ to $\alpha_{\infty}<\alpha_{\infty}-\left(1-\alpha_{\infty}\right) p /\left(N_{*}-p\right)$, which is impossible since $\alpha_{\infty}<\alpha_{1}=1$. Thus, $\alpha_{\infty}=\left(\left(N_{*}-p\right) /\left(N_{*}-\alpha_{\infty}\right)\right) \alpha_{\infty}$ and, 
as $\left.\left(N_{*}-p\right) /\left(N_{*}-\alpha_{\infty}\right) \in\right] 0,1\left[\right.$, this shows that $\alpha_{\infty}=0$. Therefore, the sequence $\left(\alpha_{n}\right)_{n \geq 1}$ tends to 0 as $n \rightarrow \infty$.

We conclude this step by proving that, for all $n \geq 2$,

$$
\bar{p}-\alpha_{n} \leq \frac{N_{*}\left(p-\alpha_{n-1}\right)}{N_{*}-p} \text { and } p-\alpha_{n} \leq \frac{N_{*}\left(p-\alpha_{n-1}\right)}{N_{*}-\alpha_{n-1}} .
$$

The first inequality of (35) is an immediate consequence of the definition of $\alpha_{n}$. To obtain the second inequality, we write $\alpha_{n} \geq$ $\left(N_{*}-p\right) \alpha_{n-1} /\left(N_{*}-\alpha_{n-1}\right)$, which implies $p-\alpha_{n} \leq\left(N_{*} p-\alpha_{n-1} p-\right.$ $\left.N_{*} \alpha_{n-1}+p \alpha_{n-1}\right) /\left(N_{*}-\alpha_{n-1}\right)=N_{*}\left(p-\alpha_{n-1}\right) /\left(N_{*}-\alpha_{n-1}\right)$.

Step 2: a set of functions satisfying (23).

Let $\varphi: \mathbb{R} \rightarrow \mathbb{R}^{+}$be defined by $\varphi(s)=\exp (-1 /(1-s))$ if $s<1$ and $\varphi(s)=0$ if $s \geq 1$; for all $m>0, \varphi^{m} \in C^{\infty}\left(\mathbb{R} ; \mathbb{R}^{+}\right)$and, for all $m \in$ ]0,1[, there exists $Q_{m}$ such that $\left|\varphi^{\prime}\right| \leq Q_{m} \varphi^{m}$ on $\mathbb{R}^{+}$(indeed, this inequality is satisfied on $\left[1, \infty\left[\right.\right.$ where $\varphi^{\prime}=0$ and, on $[0,1[$, we have $\left|\varphi^{\prime}\right| \varphi^{-m}=\exp (-(1-m) /(1-s)) /(1-s)^{2}$, which is bounded since $1-m>0)$.

If $e \in \mathbb{R}^{N}$ and $\eta>0$, we define $\theta_{e, \eta}(x)=\varphi\left(|x-e|^{2} / \eta^{2}\right)$. For all $m>0, \theta_{e, \eta}^{m}=\varphi^{m}\left(|\cdot-e|^{2} / \eta^{2}\right)$ is in $C^{\infty}\left(\mathbb{R}^{N} ; \mathbb{R}^{+}\right)$by composition. Moreover, if $m \in] 0,1[$, we have, on $B(e, \eta)$,

$$
\begin{aligned}
\left|\nabla \theta_{e, \eta}\right| & =\frac{2|\cdot-e|}{\eta^{2}}\left|\varphi^{\prime}\right|\left(\frac{|\cdot-e|^{2}}{\eta^{2}}\right) \\
& \leq \frac{2}{\eta} Q_{m} \varphi^{m}\left(\frac{|\cdot-e|^{2}}{\eta^{2}}\right) \\
& =\frac{2 Q_{m}}{\eta} \theta_{e, \eta}^{m} .
\end{aligned}
$$

Since this inequality is also satisfied outside $B(e, \eta)$ (because $\nabla \theta_{e, \eta}$ is null outside this ball), we deduce that $\left|\nabla \theta_{e, \eta}\right| \leq 2 \eta^{-1} Q_{m} \theta_{e, \eta}^{m}$ on $\mathbb{R}^{N}$.

$\theta_{e, \eta}$ being null outside $B(e, \eta)$, we conclude that, if $\bar{B}(e, \eta) \cap K=$ $\emptyset$, then $\theta_{e, \eta}$ satisfies (23).

Step 3: the bootstrap.

Let $e \in \Omega \backslash K$ and $0<\eta<\operatorname{dist}(e, K)$. Define $\eta_{n}=\eta / 2+\eta / 2^{n}$.

In this step, we want to prove by induction that, for all $n \geq 1$, for all $0<r<N_{*}\left(p-\alpha_{n}\right) /\left(N_{*}-p\right)$ and all $0<s<N_{*}\left(p-\alpha_{n}\right) /\left(N_{*}-\right.$ $\left.\alpha_{n}\right)$, there exists $C_{n, r, s}$ only depending on $(\Lambda, e, \eta, n, r, s)$ such that 


$$
\int_{\Omega \cap B\left(e, \eta_{n}\right)}\left(|u|^{r}+|\nabla u|^{s}\right) \leq C_{n, r, s} .
$$

The case $n=1$ is an immediate consequence of Theorem 1 , since $\alpha_{1}=1$

Take $n \geq 2$ and suppose that, for $0<r_{0}<N_{*}\left(p-\alpha_{n-1}\right) /\left(N_{*}-p\right)$ and $0<s_{0}<N_{*}\left(p-\alpha_{n-1}\right) /\left(N_{*}-\alpha_{n-1}\right)$, there exists $C_{n-1, r_{0}, s_{0}}$ only depending on $\left(\Lambda, e, \eta, n-1, r_{0}, s_{0}\right)$ such that

$$
\int_{\Omega \cap B\left(e, \eta_{n-1}\right)}\left(|u|^{r_{0}}+|\nabla u|^{s_{0}}\right) \leq C_{n-1, r_{0}, s_{0}} .
$$

Let $0<r<N_{*}\left(p-\alpha_{n}\right) /\left(N_{*}-p\right)$ and $0<s<N_{*}\left(p-\alpha_{n}\right) /\left(N_{*}-\right.$ $\left.\alpha_{n}\right)$; we can find $\left.\left.\gamma \in\right] \alpha_{n}, 1\right]$, only depending on $(n, r, s)$, such that $r<N_{*}(p-\gamma) /\left(N_{*}-p\right)$ and $s<N_{*}(p-\gamma) /\left(N_{*}-\gamma\right)$. Let $r_{0}=\bar{p}-$ $\gamma$ and $s_{0}=p-\gamma$; by (35), since $\gamma>\alpha_{n}$, we have $0<r_{0}<N_{*}(p-$ $\left.\alpha_{n-1}\right) /\left(N_{*}-p\right)$ and $0<s_{0}<N_{*}\left(p-\alpha_{n-1}\right) /\left(N_{*}-\alpha_{n-1}\right)$, thus, for $C_{n-1, r_{0}, s_{0}}$ as above,

$$
\begin{aligned}
\int_{\Omega \cap \bar{B}\left(e, \eta_{n-1}\right)} & \left(|u|^{\bar{p}-\gamma}+|\nabla u|^{p-\gamma}\right) \\
& =\int_{\Omega \cap B\left(e, \eta_{n-1}\right)}\left(|u|^{\bar{p}-\gamma}+|\nabla u|^{p-\gamma}\right) \leq C_{n-1, r_{0}, s_{0}}
\end{aligned}
$$

(notice that meas $\left.\left(\partial\left(B\left(e, \eta_{n-1}\right)\right)\right)=0\right)$. Since $r<N_{*}(p-\gamma) /\left(N_{*}-\right.$ $p)$ and $s<N_{*}(p-\gamma) /\left(N_{*}-\gamma\right)$, Corollary 1 applied to $\alpha=\gamma$ and $\theta=\theta_{e, \eta_{n-1}}$ (the support of which is included in $\left.\bar{B}\left(e, \eta_{n-1}\right)\right)$ gives then $C_{1}$ only depending on $\Lambda, \theta_{e, \eta_{n-1}}, C_{n-1, r_{0}, s_{0}}, \gamma, r$ and $s$, i.e. only depending on $(\Lambda, e, \eta, n, r, s)$, such that

$$
\int_{\Omega}\left(\left|\theta_{e, \eta_{n-1}} u\right|^{r}+\left|\nabla\left(\theta_{e, \eta_{n-1}} u\right)\right|^{s}\right) \leq C_{1} .
$$

Since $\theta_{e, \eta_{n-1}} \geq C_{2}>0$ on $B\left(e, \eta_{n}\right)$, where $C_{2}$ only depends on $(\eta, n)$, we deduce that

$$
\int_{\Omega \cap B\left(e, \eta_{n}\right)}|u|^{r} \leq \frac{C_{1}}{C_{2}^{r}}
$$

and that

$$
\int_{\Omega \cap B\left(e, \eta_{n}\right)}|\nabla u|^{s}
$$




$$
\begin{aligned}
& \leq \frac{1}{C_{2}^{s}} \int_{\Omega \cap B\left(e, \eta_{n}\right)}\left|\theta_{e, \eta_{n-1}} \nabla u\right|^{s} \\
& \leq \frac{1}{C_{2}^{s}} \int_{\Omega \cap B\left(e, \eta_{n}\right)} 2^{s}\left|\nabla\left(\theta_{e, \eta_{n-1}} u\right)\right|^{s}+2^{s}\left|u \nabla \theta_{e, \eta_{n-1}}\right|^{s} \\
& \leq \frac{2^{s} C_{1}}{C_{2}^{s}}+\frac{2^{s}||\left|\nabla \theta_{e, \eta_{n-1}}\right|||_{L^{\infty}\left(\mathbb{R}^{N}\right)}^{s}}{C_{2}^{s}} \int_{\Omega \cap B\left(e, \eta_{n}\right)}|u|^{s} .
\end{aligned}
$$

Since $N_{*}\left(p-\alpha_{n}\right) /\left(N_{*}-p\right) \geq N_{*}\left(p-\alpha_{n}\right) /\left(N_{*}-\alpha_{n}\right)$, we can always suppose that $r \geq s$, and we obtain thus

$$
\begin{aligned}
& \int_{\Omega \cap B\left(e, \eta_{n}\right)}|\nabla u|^{s} \\
& \leq \frac{2^{s} C_{1}}{C_{2}^{s}}+\frac{2^{s}||\left|\nabla \theta_{e, \eta_{n-1} \mid}\right| \|_{L^{\infty}\left(\mathbb{R}^{N}\right)}^{s}}{C_{2}^{s}} \int_{\Omega \cap B\left(e, \eta_{n}\right)}\left(1+|u|^{r}\right) \\
& \leq \frac{2^{s} C_{1}}{C_{2}^{s}}+\frac{2^{s}\left\|\left|\nabla \theta_{e, \eta_{n-1}}\right|\right\|_{L^{\infty}\left(\mathbb{R}^{N}\right)}^{s}}{C_{2}^{s}}\left(\operatorname{meas}(\Omega)+\frac{C_{1}}{C_{2}^{r}}\right) .
\end{aligned}
$$

(36) and (37) conclude the induction.

Step 4: conclusion.

Let $q \in\left[1, p\left[\right.\right.$; we have $N_{*} q /\left(N_{*}-q\right)<N_{*} p /\left(N_{*}-p\right)$. Since $\alpha_{n} \rightarrow$ 0 , there exists $n_{0} \geq 1$ such that $N_{*} q /\left(N_{*}-q\right)<N_{*}\left(p-\alpha_{n_{0}}\right) /\left(N_{*}-\right.$ $p)$ and $q<N_{*}\left(p-\alpha_{n_{0}}\right) /\left(N_{*}-\alpha_{n_{0}}\right)$ ( $n_{0}$ only depends on $\left.q\right)$.

Take $e \in \Omega \backslash F$; we notice that $\varepsilon<\operatorname{dist}(e, K)$. By Step 3 , there exists thus $C(e)$ only depending on $\Lambda, e, \varepsilon, n_{0}, N_{*} q /\left(N_{*}-q\right)$ and $q$, i.e. on $(\Lambda, e, \varepsilon, q)$, such that

$$
\begin{aligned}
\int_{\Omega \cap B\left(e, \frac{\varepsilon}{2}\right)} & \left(|u|^{\frac{N_{*} q}{N_{*}-q}}+|\nabla u|^{q}\right) \\
& \leq \int_{\Omega \cap B\left(e, \frac{\varepsilon}{2}+\frac{\varepsilon}{2^{n_{0}}}\right)}\left(|u|^{\frac{N_{*} q}{N_{*}-q}}+|\nabla u|^{q}\right) \\
& \leq C(e) .
\end{aligned}
$$

The compact set $\overline{\Omega \backslash F}$ is covered by $\{B(e, \varepsilon / 2), e \in \Omega \backslash F\}$ (the points of $\partial(\Omega \backslash F)$ are in the union of these balls because the radius is fixed), so we can find $\left(e_{1}, \ldots, e_{l}\right) \in \Omega \backslash F$ - only depending on $(F, \varepsilon)$, i.e. on $(K, \varepsilon)-$ such that $\Omega \backslash F \subset \cup_{i=1}^{l} B\left(e_{i}, \varepsilon / 2\right)$. Writing (38) for $e_{1}, \ldots, e_{l}$ and summing these inequalities, we find 


$$
\int_{\Omega \backslash F}\left(|u|^{\frac{N_{*} q}{N_{*}-q}}+|\nabla u|^{q}\right) \leq \sum_{i=1}^{l} C\left(e_{i}\right),
$$

which is the desired estimate.

We can now prove the local estimates theorem.

\section{Proof of Theorem 2.}

Let $\psi \in C_{c}^{\infty}\left(\mathbb{R}^{N}\right)$ be such that $\psi=1$ on a neighborhood of $K+$ $\bar{B}(0, \varepsilon / 3)$ and $\psi=0$ outside $K+B(0, \varepsilon / 2)$ (the choice of $\psi$ only depends on $(K, \varepsilon))$. We take $\theta=|1-\psi|^{\zeta}$, with $\zeta=\max (2,2 /(p-$ $1)$ ), and notice that $\theta, \theta^{p-1}$ and $\theta^{p}$ are $C^{1}$ on $\mathbb{R}^{N}$ (they all can be written as $|1-\psi|^{l}$ with $l>1$, and, if $l>1, s \rightarrow|s|^{l}$ is $C^{1}$ on $\left.\mathbb{R}\right)$. Moreover, $\theta=0$ on a neighborhood of $K$. Thus, using $\theta^{p} u$ as a test function in (8), we obtain

$$
\begin{aligned}
& \Lambda \| \theta^{p} u \|_{W_{0}^{1, p}(\Omega)} \\
& \geq\left\langle f, \theta^{p} u\right\rangle_{W^{-1, p^{\prime}}(\Omega), W_{0}^{1, p}(\Omega)} \\
&= \int_{\Omega} a(x, u, \nabla u) \cdot \nabla\left(\theta^{p} u\right)+\int_{\Omega} \Phi(x, u) \cdot \nabla\left(\theta^{p} u\right) \\
&= \int_{\Omega} \theta^{p} a(x, u, \nabla u) \cdot \nabla u+\int_{\Omega} u a(x, u, \nabla u) \nabla\left(\theta^{p}\right) \\
&+\int_{\Omega} \theta^{p} \Phi(x, u) \cdot \nabla u+\int_{\Omega} u \Phi(x, u) \cdot \nabla\left(\theta^{p}\right) \\
& \geq \nu \int_{\Omega}|\theta \nabla u|^{p}-\int_{\Omega} \theta^{p} \Theta-\int_{\Omega \backslash\left(K+\bar{B}\left(0, \frac{\varepsilon}{3}\right)\right)} \theta^{p} g\left(1+|u|^{p-1}\right)|\nabla u| \\
& \quad-\int_{\Omega \backslash\left(K+\bar{B}\left(0, \frac{\varepsilon}{3}\right)\right)}|u|\left(h+\beta|u|^{p-1}+\beta|\nabla u|^{p-1}\right. \\
&\left.\quad+g+g|u|^{p-1}\right)\left|\nabla\left(\theta^{p}\right)\right|
\end{aligned}
$$

(notice that $\nabla\left(\theta^{p}\right)$ and $\theta$ are null on $K+\bar{B}(0, \varepsilon / 3)$ ).

Since $\theta^{p-1}$ is $C^{1}$, we have

$$
\left\|\theta^{p} u\right\|_{W_{0}^{1, p}(\Omega)}=\left\|\theta^{p-1} \theta u\right\|_{W_{0}^{1, p}(\Omega)} \leq C_{1}\|\theta u\|_{W_{0}^{1, p}(\Omega)}
$$

where $C_{1}$ only depends on $\theta^{p-1}$, i.e. on $(K, \varepsilon)$.

Let $\delta>0$; take $g_{1} \in L^{\infty}(\Omega)$ and $g_{2} \in L^{N_{*} /(p-1)}(\Omega)$ such that $g=$ $g_{1}+g_{2}$ and $\left\|g_{2}\right\|_{L^{N_{*} /(p-1)}(\Omega)} \leq \delta$ (the choice of such a decomposition 
only depends on $\delta$ ). Since $\theta, g_{1}$ and $\nabla\left(\theta^{p}\right)$ are bounded on $\Omega$, we deduce from (39), by Young's inequality (and using the fact that $\bar{p} \geq p$, which implies $\left.|u|^{p} \leq 1+|u|^{\bar{p}}\right)$,

$$
\begin{aligned}
& C_{1} \Lambda\|\theta u\|_{W_{0}^{1, p}(\Omega)} \\
& \geq \nu \int_{\Omega}|\theta \nabla u|^{p}-C_{2}-\int_{\Omega \backslash\left(K+\bar{B}\left(0, \frac{\varepsilon}{3}\right)\right)} g_{2}|\theta u|^{p-1}|\theta \nabla u| \\
& -C_{2} \int_{\Omega \backslash\left(K+\bar{B}\left(0, \frac{\varepsilon}{3}\right)\right)}\left(g|\nabla u|+|u|^{p-1}|\nabla u|+h^{p^{\prime}}+g^{p^{\prime}}+|u|^{p}\right. \\
& \left.+|u|^{\bar{p}}+|\nabla u|^{p-1}|u|+g|u|^{p}\right) \\
& \geq \frac{\nu}{2} \int_{\Omega}|\theta \nabla u|^{p}-C_{3}-C_{3} \int_{\Omega} g_{2}^{p^{\prime}}|\theta u|^{p} \\
& -C_{3} \int_{\Omega \backslash\left(K+\bar{B}\left(0, \frac{\varepsilon}{3}\right)\right)}\left(g|\nabla u|+|u|^{p-1}|\nabla u|\right. \\
& \left.+|u|^{\bar{p}}+|\nabla u|^{p-1}|u|+g|u|^{p}\right)
\end{aligned}
$$

where $C_{2}, C_{3}$ only depend on $(K, \varepsilon, \delta)$ - recall that $g_{1}$ only depends on $\delta$ and that $\theta$ only depends on $(K, \varepsilon)$.

Since, by the simplified Young's inequality with $N_{*} /(p-1)>1$, we have

$$
g|\nabla u| \leq g^{\frac{N_{*}}{p-1}}+|\nabla u|^{\frac{N_{*}}{N_{*}-p+1}} \quad \text { and } \quad g|u|^{p} \leq g^{\frac{N_{*}}{p-1}}+|u|^{\frac{N_{*} p}{N_{*}-p+1}},
$$

we obtain, from (40), $C_{4}$ only depending on $(\Lambda, K, \varepsilon, \delta)$ such that

$$
\begin{aligned}
& \int_{\Omega}|\nabla(\theta u)|^{p} \\
& \leq 2^{p} \int_{\Omega}|\theta \nabla u|^{p}+2^{p} \int_{\Omega}|u \nabla \theta|^{p} \\
& \leq\left. C_{4}|| \theta u\right|_{W_{0}^{1, p}(\Omega)}+C_{4}+C_{4} \int_{\Omega} g_{2}^{p^{\prime}}|\theta u|^{p} \\
& \quad+C_{4} \int_{\Omega \backslash\left(K+\bar{B}\left(0, \frac{\varepsilon}{3}\right)\right)}\left(|\nabla u|^{\frac{N_{*}}{N_{*}-p+1}}+|u|^{\frac{N_{*} p p}{N_{*}-p+1}}+|u|^{\bar{p}}\right. \\
& \left.\quad+|\nabla u|^{p-1}|u|+|u|^{p-1}|\nabla u|\right)
\end{aligned}
$$

(we have used the fact that $\nabla \theta$ is bounded on $\Omega$ and null on $K+$ $\bar{B}(0, \varepsilon / 3)$, and we have bounded $|u|^{p}$ by $\left.1+|u|^{\bar{p}}\right)$. 
Let $q \in] \max (p-1,1), p[$. The simplified Young's inequality (with $q /(p-1)>1$ and $q)$ give

$$
\begin{aligned}
& |\nabla u|^{p-1}|u| \leq|\nabla u|^{q}+|u|^{\frac{q}{q-p+1}} \\
& \text { and } \quad|u|^{p-1}|\nabla u| \leq|u|^{\frac{q(p-1)}{q-1}}+|\nabla u|^{q} \text {. }
\end{aligned}
$$

Moreover, by Hölder's inequality (with $N_{*} / p$ ) and the Sobolev injections,

$$
\begin{aligned}
\int_{\Omega} g_{2}^{p^{\prime}}|\theta u|^{p} & \leq\left\|\left.g_{2}\right|_{L^{\frac{N_{*}}{p-1}}(\Omega)} ^{p^{\prime}}\right\| \theta u \|_{L^{\frac{N_{*} p}{N *}}(\Omega)}^{p} \\
& \leq C_{5} \delta^{p^{\prime}}\||\nabla(\theta u)|\|_{L^{p}(\Omega)}^{p} .
\end{aligned}
$$

Setting $\delta>0$ such that $1-1 / p-C_{5} \delta^{p^{\prime}}>0$ and writing

$$
C_{4}\|\theta u\|_{W_{0}^{1, p}(\Omega)} \leq \frac{1}{p^{\prime}} C_{4}^{p^{\prime}}+\frac{1}{p}\||\nabla(\theta u)|\|_{L^{p}(\Omega)}^{p},
$$

(43) and (42) injected in (41) give $C_{6}$ only depending on $(\Lambda, K, \varepsilon)$ such that, for all $q \in] \max (p-1,1), p[$,

$$
\begin{aligned}
& \int_{\Omega}|\nabla(\theta u)|^{p} \\
& \leq C_{6}+C_{6} \int_{\Omega \backslash\left(K+\bar{B}\left(0, \frac{\varepsilon}{3}\right)\right)}\left(|u|^{\bar{p}}+|u|^{\frac{N_{*} p}{N_{*}-p+1}}+|u|^{\frac{q}{q-p+1}}\right. \\
& \left.+|u|^{\frac{q(p-1)}{q-1}}+|\nabla u|^{\frac{N_{*}}{N_{*}-p+1}}+|\nabla u|^{q}\right) .
\end{aligned}
$$

We notice that

$$
\begin{aligned}
& \bar{p}<\frac{\left(N_{*}-1\right) p}{N_{*}-p}<\frac{N_{*} p}{N_{*}-p}, \\
& \frac{N_{*} p}{N_{*}-p+1}<\frac{N_{*} p}{N_{*}-p} \\
\text { and } \quad & \frac{N_{*}}{N_{*}-p+1}<p
\end{aligned}
$$

(the last inequality comes down to $\left(p-N_{*}\right)(p-1)<0$, which is true since $p \in] 1, N_{*}[$ ). Moreover, as $q \rightarrow p$, we have

$$
\frac{q}{q-p+1} \rightarrow p<\frac{N_{*} p}{N_{*}-p},
$$




$$
\begin{aligned}
\frac{q(p-1)}{q-1} & \rightarrow p<\frac{N_{*} p}{N_{*}-p} \\
\text { and } \quad \frac{N_{*} q}{N_{*}-q} & \rightarrow \frac{N_{*} p}{N_{*}-p} .
\end{aligned}
$$

We can thus find $q \in] \max (p-1,1), p[$ such that

$$
\begin{aligned}
& \bar{p} \leq \frac{N_{*} q}{N_{*}-q}, \quad \frac{N_{*} p}{N_{*}-p+1} \leq \frac{N_{*} q}{N_{*}-q}, \\
& \frac{N_{*}}{N_{*}-p+1} \leq q, \quad \frac{q}{q-p+1} \leq \frac{N_{*} q}{N_{*}-q} \\
& \text { and } \quad \frac{q(p-1)}{q-1} \leq \frac{N_{*} q}{N_{*}-q} .
\end{aligned}
$$

Applying then Proposition 4 to this $q$ (and with $\varepsilon / 3$ instead of $\varepsilon$ ), we find $C_{7}$ only depending on $(\Lambda, K, \varepsilon)$ such that

$$
\begin{aligned}
\int_{\Omega \backslash\left(K+\bar{B}\left(0, \frac{\varepsilon}{3}\right)\right)} & \left(|u|^{\bar{p}}+|u|^{\frac{N_{*} p}{N_{*}-p+1}}+|u|^{\frac{q}{q-p+1}}+|u|^{\frac{q(p-1)}{q-1}}\right) \\
& \leq 4 \int_{\Omega \backslash\left(K+\bar{B}\left(0, \frac{\varepsilon}{3}\right)\right)}\left(1+|u|^{\frac{N_{*} q}{N_{*}-q}}\right) \\
& \leq 4 \operatorname{meas}(\Omega)+4 C_{7},
\end{aligned}
$$

and

$$
\begin{aligned}
\int_{\Omega \backslash\left(K+\bar{B}\left(0, \frac{\varepsilon}{3}\right)\right)} & \left(|\nabla u|^{\frac{N_{*}}{N_{*}-p+1}}+|\nabla u|^{q}\right) \\
& \leq \int_{\Omega \backslash\left(K+\bar{B}\left(0, \frac{\varepsilon}{3}\right)\right)}\left(1+2|\nabla u|^{q}\right) \\
& \leq \operatorname{meas}(\Omega)+2 C_{7} .
\end{aligned}
$$

Returning to (44), we obtain

$$
\int_{\Omega}|\nabla(\theta u)|^{p} \leq C_{6}+C_{6}\left(5 \operatorname{meas}(\Omega)+6 C_{7}\right):=C_{8}
$$

with $C_{8}$ only depending on $(\Lambda, K, \varepsilon)$, that is to say $\|\theta u\|_{W_{0}^{1, p}(\Omega)} \leq$ $C_{8}^{1 / p}$. By the Sobolev injection, we get $\|\theta u\|_{L^{p^{*}}(\Omega)} \leq C_{9} C_{8}^{1 / p}$. Since $\theta \equiv 1$ on the open set $\Omega \backslash(K+\bar{B}(0, \varepsilon))$, these last two estimates give the result of the theorem. 


\section{Existence and regularity result for an equation with measure data}

We can now prove Theorem 3. In fact, thanks to the previous estimates and to the technique of [4], the proof is very simple.

\section{Proof of Theorem 3}

We can find $\left(\mu_{n}\right)_{n \geq 1} \in W^{-1, p^{\prime}}(\Omega) \cap \mathcal{M}(\Omega)$ converging weakly-* to $\mu$ in $\mathcal{M}(\Omega)$ and such that, for all $\varepsilon>0, \operatorname{supp}\left(\mu_{n}\right) \subset \operatorname{supp}(\mu)+B(0, \varepsilon)$ for $n$ large enough (in fact, most of the classical ways to approximate $\mu$ by regular data - for example through a convolution method, or by discretizing $\mu$ on a grid - satisfy this property on the supports of the approximations). Take $u_{n}$ a solution to (8) with $\mu_{n}$ instead of $\mu$.

Since $\left(\mu_{n}\right)_{n \geq 1}$ converges in $\mathcal{M}(\Omega)$ weak-*, it is bounded in this space; thus, by Theorem 1 ,

$$
\left(u_{n}\right)_{n \geq 1} \text { is bounded in } W_{0}^{1, q}(\Omega) \text { for all } q<\frac{N_{*}(p-1)}{N_{*}-1}
$$

(notice that, as $p>2-1 / N_{*}$, we have $N_{*}(p-1) /\left(N_{*}-1\right)>1$ ). Moreover, for all $\varepsilon>0$, denoting $K=\overline{\operatorname{supp}(\mu)+B(0, \varepsilon / 2)}$, the support of $\mu_{n}$ is contained in $K$ for $n$ large enough; by Theorem 2, $\left(u_{n}\right)_{n \geq 1}$ is thus bounded in $L^{p^{*}}\left(\Omega \backslash(K+\bar{B}(0, \varepsilon / 2)) \cap W^{1, p}(\Omega \backslash(K+\right.$ $\bar{B}(0, \varepsilon / 2))$; since $K+\bar{B}(0, \varepsilon / 2) \subset F_{\varepsilon}:=\overline{\operatorname{supp}(\mu)+B(0, \varepsilon)}$, this implies that

$$
\begin{aligned}
\text { for all } \varepsilon>0, & \left(u_{n}\right)_{n \geq 1} \text { is bounded in } L^{p^{*}}\left(\Omega \backslash F_{\varepsilon}\right) \\
& \text { and in } W^{1, p}\left(\Omega \backslash F_{\varepsilon}\right) .
\end{aligned}
$$

By (45), (46), a diagonal process and Rellich's theorem, we can extract a sequence of $\left(u_{n}\right)_{n \geq 1}$, still denoted $\left(u_{n}\right)_{n \geq 1}$, such that $u_{n} \rightarrow$ $u$ a.e. on $\Omega$, weakly in $W_{0}^{1, q}(\Omega)$ for all $q<N_{*}(p-1) /\left(N_{*}-1\right)$ and, for all $\varepsilon>0$, weakly in $L^{p^{*}}\left(\Omega \backslash F_{\varepsilon}\right)$ and in $W^{1, p}\left(\Omega \backslash F_{\varepsilon}\right)$.

Using then the technique of [4], we can prove that $\nabla u_{n} \rightarrow \nabla u$ a.e. on $\Omega$ (there is no $\Phi$ in [4], but the technique to prove the a.e. convergence of the gradients works fine even with this additional term). This allows to pass to the limit in the equation satisfied by $u_{n}$ and to see that $u$ is a solution to (9).

If $p \leq 2-1 / N_{*}$ (in this case, $N_{*}=N$ ), it is well-known that a 
solution to (1) with $\mu$ measure is not to be sought in a Sobolev space (one can notice that, in this case, Theorem 1 does not give an estimate in a Sobolev space). To solve this problem, two main notions of solutions have been introduced: entropy solutions (see [2]) or renormalized solutions (see [7]).

For each of these notions, the existence of a solution is proved thanks to an approximation method; thus, Theorem 2 also allows to obtain entropy or renormalized solutions with better local regularity results than usual. For example, under Hypotheses (2)-(6), if $\mu \in L^{1}(\Omega)$ and $f \in W^{-1, p^{\prime}}(\Omega)$, the technique of [2], associated to Estimate (22) and to Theorem 2, allows to prove the existence of a solution to (1) in the sense:

$$
\left\{\begin{array}{l}
u: \Omega \rightarrow \mathbb{R} \text { is a measurable function, } \\
\forall k \geq 0, T_{k}(u) \in W_{0}^{1, p}(\Omega), \\
\forall \varepsilon>0, \text { denoting } F_{\varepsilon}=\overline{\operatorname{supp}(\mu)+B(0, \varepsilon)} \\
\quad u \in L^{p^{*}}\left(\Omega \backslash F_{\varepsilon}\right) \cap W^{1, p}\left(\Omega \backslash F_{\varepsilon}\right), \\
\int_{\Omega} a(x, u, \nabla u) \cdot \nabla\left(T_{k}(u-\varphi)\right) \\
+\int_{\Omega} \Phi(x, u) \cdot \nabla\left(T_{k}(u-\varphi)\right) \\
\quad=\int_{\Omega} \mu T_{k}(u-\varphi)+\left\langle f, T_{k}(u-\varphi)\right\rangle_{W^{-1, p^{\prime}}(\Omega), W_{0}^{1, p}(\Omega)} \\
\forall \varphi \in W_{0}^{1, p}(\Omega) \cap L^{\infty}(\Omega) .
\end{array}\right.
$$

\section{Appendix}

The first lemma is a well-known result concerning the integrability properties of functions in Marcinkiewicz spaces.

Lemma 1 Let $v: \Omega \rightarrow \mathbb{R}$ be a measurable function and $r>0$. Suppose that there exists $M$ such that

for all $k \geq 1, \operatorname{meas}(\{|v| \geq k\}) \leq M k^{-r}$.

Then, for all $s \in] 0, r[$, there exists $C$ only depending on $(\Omega, M, r, s)$ 
such that

$$
\int_{\Omega}|v|^{s} \leq C
$$

\section{Proof of Lemma 1}

We know (this is a simple application of the Fubini-Tonelli theorem) that

$$
\int_{\Omega}|v|^{s}=\int_{0}^{\infty} \operatorname{meas}\left(\left\{|v|^{s} \geq t\right\}\right) d t .
$$

Bounding meas $\left(\left\{|v|^{s} \geq t\right\}\right)$ by meas $(\Omega)$ if $t \leq 1$ and by $M t^{-r / s}$ if $t \geq 1$ (because $\left\{|v|^{s} \geq t\right\}=\left\{|v| \geq t^{1 / s}\right\}$ ), and using the fact that $r / s>1$, we find

$$
\int_{\Omega}|v|^{s} \leq \operatorname{meas}(\Omega)+M \int_{1}^{\infty} t^{-r / s} d t=\operatorname{meas}(\Omega)+\frac{M}{\frac{r}{s}-1},
$$

which concludes the proof.

The following result is a very simple generalization of a lemma in $[2]$.

Lemma 2 Let $\alpha \in] 0, p\left[\right.$ and $v \in W_{0}^{1, p}(\Omega)$. If there exists $M$ such that, for all $k \geq 1$,

$$
\int_{\Omega}\left|\nabla\left(T_{k}(v)\right)\right|^{p} \leq M k^{\alpha},
$$

then there exists $C$ only depending on $\left(\Omega, p, N_{*}, M\right)$ such that, for all $k>0$,

$$
\begin{array}{ll} 
& \operatorname{meas}(\{|v| \geq k\}) \leq C k^{-\frac{N_{*}(p-\alpha)}{N_{*}-p}} \\
\text { and } \quad & \operatorname{meas}(\{|\nabla v| \geq k\}) \leq C k^{-\frac{N_{*}(p-\alpha)}{N_{*}-\alpha}} .
\end{array}
$$

\section{Proof of Lemma 2.}

Let $k \geq 1$. Thanks to the Sobolev injection, we can find $C_{1}$ only depending on $\left(\Omega, p, N_{*}\right)$ such that

$$
\left\|T_{k}(v)\right\|_{L^{p^{*}}(\Omega)} \leq C_{1}\left\|\left|\nabla\left(T_{k}(v)\right)\right|\right\|_{L^{p}(\Omega)} \leq C_{1} M^{\frac{1}{p}} k^{\frac{\alpha}{p}} .
$$

Since 


$$
\operatorname{meas}(\{|v| \geq k\})=\operatorname{meas}\left(\left\{\left|T_{k}(v)\right| \geq k\right\}\right) \leq\left. k^{-p^{*}}|| T_{k}(v)\right|_{L^{p^{*}}(\Omega)} ^{p^{*}},
$$

we deduce that meas $(\{|v| \geq k\}) \leq C_{1}^{p^{*}} M^{p^{*} / p} k^{-p^{*}} k^{p^{*} \alpha / p}$; this concludes the first estimate for $k \geq 1$, as

$$
p^{*}\left(1-\frac{\alpha}{p}\right)=\frac{N_{*}(p-\alpha)}{N_{*}-p} .
$$

If $k \leq 1$, we simply write

$$
\operatorname{meas}(\{|v| \geq k\}) \leq \operatorname{meas}(\Omega) \leq \operatorname{meas}(\Omega) k^{-\frac{N_{*}(p-\alpha)}{N_{*}-p}} .
$$

Let $k \geq 1$ and take $\lambda \geq 1$. We have

$$
\begin{aligned}
\{|\nabla v| \geq k\} & \subset\{|\nabla v| \geq k,|v| \geq \lambda\} \cup\{|\nabla v| \geq k,|v| \leq \lambda\} \\
& \subset\{|v| \geq \lambda\} \cup\left\{\left|\nabla\left(T_{\lambda}(v)\right)\right| \geq k\right\} \cup A,
\end{aligned}
$$

where meas $(A)=0$ (we use $\nabla\left(T_{\lambda}(v)\right)=\mathbf{1}_{\{|v| \leq \lambda\}} \nabla v$ a.e. on $\Omega$ ). Thus, using the first estimate and the hypothesis of the lemma,

$$
\begin{aligned}
\operatorname{meas}(\{|\nabla v| \geq k\}) & \leq C_{2} \lambda^{-\frac{N_{*}(p-\alpha)}{N_{*}-p}}+k^{-p} \int_{\Omega}\left|\nabla\left(T_{\lambda}(v)\right)\right|^{p} \\
& \leq C_{2} \lambda^{-\frac{N_{*}(p-\alpha)}{N_{*}-p}}+M \lambda^{\alpha} k^{-p}
\end{aligned}
$$

where $C_{2}$ only depends on $\left(\Omega, p, N_{*}, M\right)$.

Choose now $\lambda=k^{p /\left(p_{1}+\alpha\right)}$, where $p_{1}=N_{*}(p-\alpha) /\left(N_{*}-p\right)$ (this choice comes down to taking $\lambda=k^{\beta}$ for some $\beta$ such that the powers of $k$ in the right-hand side of (48) are the same; this also comes down - up to a multiplicative constant - to minimizing the right-hand side of (48) on $\lambda$ ). We have $\lambda \geq 1$ and

$$
\lambda^{-\frac{N_{*}(p-\alpha)}{N_{*}-p}}=k^{-\frac{p p_{1}}{p_{1}+\alpha}} \quad \text { and } \quad \lambda^{\alpha} k^{-p}=k^{-\left(p-\frac{\alpha p}{p_{1}+\alpha}\right)}=k^{-\frac{p p_{1}}{p_{1}+\alpha}} .
$$

Since

$$
\frac{p p_{1}}{p_{1}+\alpha}=\frac{p N_{*}(p-\alpha)}{N_{*}(p-\alpha)+\alpha\left(N_{*}-p\right)}=\frac{N_{*}(p-\alpha)}{N_{*}-\alpha},
$$

(48) concludes the second inequality if $k \geq 1$; for $k \leq 1$, we simply bound meas $(\{|\nabla v| \geq k\})$ by meas $(\Omega) \leq \operatorname{meas}(\Omega) k^{-N_{*}(p-\alpha) /\left(N_{*}-\alpha\right)}$. 


\section{References}

1. Adams R.A. Sobolev Spaces; Academic Press; 1975.

2. Benilan P.; Boccardo L.; Gallouët T.; Gariepy R.; Pierre M.; Vazquez J. L.; An $L^{1}$-Theory of Existence and Uniqueness of Solutions of Nonlinear Elliptic Equations. Ann. Scuola Norm. Sup. Pisa Sci. Fis. Mat. 1995, IV, 22 (2).

3. Boccardo L.; Gallouët T. Nonlinear elliptic and parabolic equations involving measure data. J. Funct. Anal. 1989, 87, 241-273.

4. Boccardo L.; Gallouët T. Nonlinear elliptic equations with right-hand side measures. Comm. Partial Differential Equations 1992, 17, 641-655.

5. Boccardo L.; Gallouët T.; Orsina L. Existence and uniqueness of entropy solutions for nonlinear elliptic equations with measure data. Ann. Inst. H. Poincaré Anal. Non Linéaire 1996, 13, 539551.

6. Boccardo L.; Gallouët T.; Vazquez J-L. Nonlinear elliptic equations in $\mathbb{R}^{N}$ without growth restrictions on the data. Jour. Diff. Eqns. 1993, 105, 334-363.

7. Dal Maso G.; Murat F.; Orsina L.; Prignet A. Renormalized solutions for elliptic equations with general measure data. Ann. Scuola Norm. Sup. Pisa Cl. Sci. 1999, 28 (4), 741-808.

8. Droniou J. Non Coercive Linear Elliptic Problems. Potential Anal. 2002, 17 (2), 181-203.

9. Droniou J. PhD Thesis, CMI, Université de Provence. Available at http://www-gm3. univ-mrs.fr/ droniou/these.

10. Guibe O.; Ben Cheikh M. Résultats d'existence et d'unicité pour une classe de problèmes non linéaires et non coercitifs. C. R. Acad. Sci. Paris Sér. I Math. 1999, 329 (11), 967-972.

11. Leray J.; Lions J.L. Quelques résultats de Višik sur les problèmes elliptiques semi-linéaires par les méthodes de Minty et Browder. Bull. Soc. Math. France 1965, 93, 97-107. 